\title{
Kählerness of moduli spaces of stable sheaves over non-projective K3 surfaces
}

\author{
Arvid Perego
}

\begin{abstract}
We show that a moduli space of slope-stable coherent sheaves over a K3 surface is a compact hyperkähler manifold if and only if its second Betti number is the sum of its Hodge numbers $h^{2,0}, h^{1,1}$ and $h^{0,2}$.
\end{abstract}

\section{Introduction}

Compact hyperkähler manifolds are compact, connected Kähler manifolds which are simply connected and holomorphically symplectic and have $h^{2,0}=1$. Very few examples of them are currently known, and all the known deformation classes arise from moduli spaces of semistable coherent sheaves on a projective K3 surface or on an Abelian surface. In [PT17], we showed that if $S$ is any K3 surface, the moduli space $M_{v}(S, \omega)$ of $\mu_{\omega}$-stable coherent sheaves on $S$ of Mukai vector $v=(r, \xi, a) \in H^{2 *}(S, \mathbb{Z})$ is a compact, connected complex manifold, it carries a holomorphic symplectic form, and it is of $\mathrm{K}^{[n]}$-type (that is, it is deformation equivalent to a Hilbert scheme of points on a projective K3 surface). This holds under some hypotheses on $\omega$ and $v$ (namely, $\omega$ is a $v$-generic Kähler class, and $r$ and $\xi$ have to be relatively prime: we refer the reader to [PT17, Section 2.2] for the definition of $v$-genericity).

The main open question about these moduli spaces is if they carry a Kähler metric: if it is so, it follows that they are all compact hyperkähler manifolds of $\mathrm{K} 3^{[n]}$-type. The answer to this question is affirmative in at least three cases: when $S$ is projective, when $M_{v}(S, \omega)$ is a surface, when $M_{v}(S, \omega)$ parametrizes only locally free coherent sheaves. This led us to the following.

Conjecture 1.1. The moduli spaces $M_{v}(S, \omega)$ are Kähler manifolds.

Evidence is provided by the previous examples, where the moduli spaces are indeed Kähler, and by the fact that their geometry is somehow similar to that of a compact hyperkähler manifold; in [PT17], we show that on their second integral cohomology, there is a non-degenerate quadratic form defined as the Beauville-Bogomolov form of compact hyperkähler manifolds. But still, this analogy is not sufficient to guarantee that the moduli spaces are Kähler: it is known since [Gua94, Gua95a, Gua95b] that there are examples of compact, simply connected, holomorphically symplectic manifolds having $h^{2,0}=1$ which are not Kähler, but whose second integral cohomology carries a non-degenerate quadratic form, and for which the local Torelli theorem holds.

Received 7 May 2017, accepted in final form 5 April 2018.

2010 Mathematics Subject Classification 14D20, 32G13, 53C26.

Keywords: moduli spaces of sheaves, compact hyperkähler manifolds, K3 surfaces.

This journal is (C) Foundation Compositio Mathematica 2019. This article is distributed with Open Access under the terms of the Creative Commons Attribution Non-Commercial License, which permits non-commercial reuse, distribution, and reproduction in any medium, provided that the original work is properly cited. For commercial re-use, please contact the Foundation Compositio Mathematica. 


\section{A. Perego}

The aim of this paper is to show that the previous conjecture holds true under some additional hypothesis on the second Betti number of $M_{v}(S, \omega)$.

\subsection{Main definitions and notation}

In this section, we collect all the definitions and notation we will use in what follows.

Definition 1.2. A holomorphically symplectic manifold is a complex manifold which carries an everywhere non-degenerate holomorphic closed 2-form (called a holomorphic symplectic form).

We notice that a compact holomorphically symplectic manifold is always of even complex dimension, and a holomorphic symplectic form $\sigma$ defines an isomorphism $\sigma: T_{X} \longrightarrow \Omega_{X}$ of vector bundles, where $T_{X}$ is the tangent bundle of $X$ and $\Omega_{X}$ is the cotangent bundle of $X$ (that is, the dual bundle of $T_{X}$ ).

Let $X$ be a compact, connected complex manifold of complex dimension $d$, and $k \in\{0, \ldots, 2 d\}$ and $p, q \in \mathbb{N}$.

Definition 1.3. The $k$ th Betti number of $X$ is

$$
b_{k}(X)=\operatorname{dim}_{\mathbb{C}} H^{k}(X, \mathbb{C}),
$$

and the type $(p, q)$ Hodge number of $X$ is

$$
h^{p, q}(X)=\operatorname{dim}_{\mathbb{C}} H_{\bar{\partial}}^{p, q}(X) .
$$

Equivalently, one can define $h^{p, q}(X)$ to be the complex dimension of the space $H^{p}\left(X, \Omega_{X}^{q}\right)$. The general relation between the Betti and the Hodge numbers of $X$ is that

$$
b_{k}(X) \leqslant \sum_{p+q=k} h^{p, q}(X)
$$

for every $0 \leqslant k \leqslant 2 d$, and the equality holds for every $k$ if and only if the Frölicher spectral sequence of $X$ degenerates at the $E_{1}$ level.

Definition 1.4. A compact, connected complex manifold $X$ is a $b_{2}$-manifold if

$$
b_{2}(X)=h^{2,0}(X)+h^{1,1}(X)+h^{0,2}(X) .
$$

Remark 1.5. A compact, connected complex manifold $X$ is a $b_{2}$-manifold if and only if there is a weak Hodge decomposition on $H^{2}(X, \mathbb{C})$, that is, an isomorphism $H^{2}(X, \mathbb{C}) \simeq H_{\bar{\partial}}^{2,0}(X) \oplus$ $H_{\bar{\partial}}^{1,1}(X) \oplus H_{\bar{\partial}}^{0,2}(X)$. In terms of forms, this means that if $p+q=2$ and $a \in H_{\bar{\partial}}^{p, q}(X)$ is a Dolbeault class, then there is a $d$-closed 2-form $\alpha=\alpha^{p, q}+\alpha^{p+1, q-1}+\cdots+\alpha^{2,0}$ (in the Hodge-Frölicher filtration on the total de Rham complex of $X$ ) such that the Dolbeault class of $\alpha^{p, q}$ is $a$.

All compact, connected Kähler manifolds are $b_{2}$-manifolds since on a compact Kähler manifold we have a (strong) Hodge decomposition. The converse is not true in general: every compact complex surface is a $b_{2}$-manifold (since the Frölicher spectral sequence degenerates at the $E_{1}$ level), but there are several compact complex surfaces which are not Kähler.

Remark 1.6. If $X$ is a $b_{2}$-manifold, then all holomorphic 2-forms are $d$-closed. Indeed, if $\sigma$ is a holomorphic 2 -form on $X$, consider the spectral sequence morphism $d_{1}: E_{1}^{2,0} \longrightarrow E_{1}^{3,0}$. Here we have $E_{1}^{p, 0}=H_{\bar{\partial}}^{p, 0}(X)$, and $d_{1}$ sends the $\bar{\partial}$-cohomology class of $\sigma$ to the $\bar{\partial}$-cohomology class of $\partial \sigma$. As $X$ is a $b_{2}$-manifold, the morphism $d_{1}$ is trivial; hence, $\partial \sigma \in \operatorname{Im}(\bar{\partial})$. This implies that $\partial \sigma=0$, and as $\sigma$ is holomorphic, we finally get that $d \sigma=0$. 


\section{KÄHLERNESS OF MODULI SPACES}

Definition 1.7. A compact, connected complex manifold $X$ is in the Fujiki class $\mathcal{C}$ if it is bimeromorphic to a compact Kähler manifold.

Among all manifolds in the Fujiki class $\mathcal{C}$, we clearly have compact Kähler manifolds, but there are examples of manifolds in the Fujiki class $\mathcal{C}$ which are not Kähler (see, for example, [Poo86]).

Definition 1.8. A compact, connected complex manifold $X$ verifies the $\partial \bar{\partial}$-lemma for 2 -forms if every $\partial$-closed, $\bar{\partial}$-closed, $d$-exact 2 -form is $\partial \bar{\partial}$-exact.

In other terms, a compact, connected complex manifold $X$ verifies the $\partial \bar{\partial}$-lemma for 2 -forms if $\operatorname{ker}(\partial) \cap \operatorname{ker}(\bar{\partial}) \cap \operatorname{Im}(d)=\operatorname{Im}(\partial \bar{\partial})$. Another equivalent way of expressing this is that the canonical morphism $H_{B C}^{p, q}(X) \longrightarrow H^{2}(X, \mathbb{C})$ is injective for $p+q=2$ (here $H_{B C}^{p, q}(X)$ is the type $(p, q)$ Bott-Chern cohomology of $X$; that is, $\left.H_{B C}^{p, q}(X)=\operatorname{ker}(\partial) \cap \operatorname{ker} \bar{\partial} / \operatorname{Im}(\partial \bar{\partial})\right)$.

If $X$ is a compact Kähler manifold, then $X$ verifies the $\partial \bar{\partial}$-lemma for 2 -forms (more generally, for all forms; see, for example, [DGMS75, Lemma 5.11]). Moreover, if $f: X \longrightarrow Y$ is a bimeromorphic map between two compact complex manifolds and if the $\partial \bar{\partial}$-lemma for 2 -forms holds for $Y$, then it holds for $X$ (see, for example, [DGMS75, Theorem 5.22] and [Del68, Sections 4 and 5]). In particular, every compact complex manifold in the Fujiki class $\mathcal{C}$ verifies the $\partial \bar{\partial}$-lemma for 2 -forms. Moreover, if $X$ verifies the $\partial \bar{\partial}$-lemma for 2 -forms, then we have a Hodge decomposition on $H^{2}(X, \mathbb{C})$, so $X$ is a $b_{2}$-manifold (see [DGMS75, Section 5.21]).

Remark 1.9. A compact, connected complex manifold $X$ verifies the $\partial \bar{\partial}$-lemma for 2 -forms if and only if there is a strong Hodge decomposition on $H^{2}(X, \mathbb{C})$. In terms of forms, this means that if $p+q=2$ and $a \in H_{\bar{\partial}}^{p, q}(X)$ is a Dolbeault class, then there is a $d$-closed 2-form of pure type $(p, q)$ whose Dolbeault class is $a$.

If $f: \mathcal{X} \longrightarrow B$ is a holomorphic fibration, then for every $b \in B$, we let $X_{b}:=f^{-1}(b)$. Let $X$ be a compact, connected complex manifold and $B$ a connected complex manifold.

Definition 1.10. A deformation of $X$ along $B$ is a smooth and proper holomorphic family $f: \mathcal{X} \longrightarrow B$ for which there is a $0 \in B$ such that $X_{0}$ is biholomorphic to $X$.

Let now $\mathcal{P}$ be a property of complex manifolds. We recall that the analytic Zariski topology on a complex manifold $X$ is the topology whose open subsets are the complements of closed analytic subvarieties of $X$.

DeFinition 1.11. We say that the property $\mathcal{P}$ is open in the Euclidean topology (respectively, in the analytic Zariski topology) if for every deformation $\mathcal{X}$ along a connected complex manifold $B$, the set of those $b \in B$ such that $X_{b}$ verifies $\mathcal{P}$ is a Euclidean (respectively, analytic Zariski) open subset of $B$.

Kählerness is an open property in the Euclidean topology (see [KS60, Theorem 15]), but in general it is not closed (as can be seen in an example due to Hironaka in [Hir62]). It is conjectured in [DP04] that it is an open property in the analytic Zariski topology. Being in the Fujiki class $\mathcal{C}$ is not an open property in general, as can be seen in an example of Campana in [Cam91], but it is conjectured to be closed (see [Pop14]). Verifying the $\partial \bar{\partial}$-lemma for 2 -forms is an open property in the Euclidean topology (see the Ph.D. thesis of C.-C. Wu, Harvard University, 2006, or [AT13, Corollary 2.7]). Being a $b_{2}$-manifold is open in the analytic Zariski topology (as the Hodge numbers are upper semi-continuous in the analytic Zariski topology; see [Dem95] and references therein). 


\section{A. Perego}

The last definitions we need are the following.

DeFinition 1.12. Let $X$ be a compact, connected complex manifold.

(i) The manifold $X$ is compact hyperkähler if it is a Kähler manifold which is simply connected and holomorphically symplectic and $h^{2,0}(X)=1$.

(ii) The manifold $X$ is deformation equivalent to a compact hyperkähler manifold if there exist a connected complex manifold $B$ and a deformation $\mathcal{X} \longrightarrow B$ of $X$ along $B$ for which there is a $b \in B$ such that $X_{b}$ is a compact hyperkähler manifold.

(iii) The manifold $X$ is a limit of compact hyperkähler manifolds if there exist a smooth and proper family $\mathcal{X} \longrightarrow B$ along a smooth connected base $B$ and a sequence $\left\{b_{n}\right\}$ of points of $B$ converging to 0 such that $X_{b_{n}}$ is a compact hyperkähler manifold.

\subsection{Main results and structure of the paper}

In this section, we let $S$ be a K3 surface. If $r \in H^{0}(S, \mathbb{Z}) \simeq \mathbb{Z}$ and $\xi \in \mathrm{NS}(S)$, we say that $r$ and $\xi$ are relatively prime when the following property is verified: if $s \in \mathbb{Z}$ is such that $r=s r^{\prime}$ and $\xi=s \xi^{\prime}$ for some $r^{\prime} \in \mathbb{Z}$ and $\xi^{\prime} \in \mathrm{NS}(S)$, then $s= \pm 1$. A Kähler class $\omega$ on $S$ will be called a polarization, and we will usually choose it to be $v$-generic (for a definition of $v$-genericity, see [PT17, Section 2.2]).

If $v=(r, \xi, a) \in H^{2 *}(S, \mathbb{Z})$, we let $v^{2}:=\xi^{2}-2 r a$; this defines a non-degenerate quadratic form on $H^{2 *}(S, \mathbb{Z})$, called a Mukai form, whose corresponding non-degenerate symmetric bilinear pairing is called a Mukai pairing. For every $v, w \in H^{2 *}(S, \mathbb{Z})$, we let $(v, w)_{\text {Muk }}$ be the value of the Mukai pairing on $v$ and $w$ : if $v=(r, \xi, a)$ and $w=(s, \zeta, b)$, then

$$
(v, w)_{\mathrm{Muk}}:=\xi \cdot \zeta-r a-s b,
$$

where $\xi \cdot \zeta$ is the intersection product on $S$. This allows us to consider

$$
v^{\perp}:=\left\{w \in H^{2 *}(S, \mathbb{Z}) \mid(v, w)_{\mathrm{Muk}}=0\right\},
$$

which is a submodule of $H^{2 *}(S, \mathbb{Z})$ (and hence has a non-degenerate quadratic form obtained by restricting the Mukai form to $\left.v^{\perp}\right)$.

The main result of the paper is the following.

Theorem 1.13. Let $S$ be a K3 surface and $\omega$ a Kähler class on $S$. We let $v=(r, \xi, a) \in H^{2 *}(S, \mathbb{Z})$ be such that $r>0$ and $\xi \in \mathrm{NS}(S)$. Suppose that $r$ and $\xi$ are relatively prime and that $\omega$ is $v$ generic. Then the moduli space $M=M_{v}(S, \omega)$ of $\mu_{\omega}$-stable coherent sheaves on $S$ with Mukai vector $v$ is Kähler if and only if it is a $b_{2}$-manifold.

As already seen before, if $M_{v}(S, \omega)$ is Kähler, then it is a $b_{2}$-manifold. The proof of the opposite implication is the content of the present paper. The hypothesis about $M_{v}(S, \omega)$ being a $b_{2}$-manifold is the weakest one under which we are able to let the strategy of our proof work. Theorem 1.13 proves Conjecture 1.1 under the additional hypothesis of the moduli spaces being $b_{2}$-manifolds. Hence, we could possibly modify Conjecture 1.1 as follows.

Conjecture 1.14. Under the hypotheses of Theorem 1.13, the moduli spaces $M_{v}(S, \omega)$ are $b_{2}$-manifolds.

Theorem 1.13 has the following immediate corollary.

Corollary 1.15. Let $\mathcal{M} \longrightarrow B$ be any smooth and proper family of moduli spaces of coherent sheaves verifying the conditions of Theorem 1.13. The set of $b \in B$ such that $M_{b}$ is Kähler is an open subset of $B$ with respect to the analytic Zariski topology. 


\section{KÄHLERNESS OF MODULI SPACES}

In view of [PT17, Theorems 1.1 and 1.2], another immediate corollary is the following.

Corollary 1.16. Let $S$ be a K3 surface and $\omega$ a Kähler class on $S$, and let $v=(r, \xi, a) \in$ $H^{2 *}(S, \mathbb{Z})$ be such that $r>0$ and $\xi \in \mathrm{NS}(S)$. Suppose that $r$ and $\xi$ are relatively prime, that $\omega$ is $v$-generic and that $M_{v}(S, \omega)$ is a $b_{2}$-manifold.

(i) The moduli space $M_{v}(S, \omega)$ is a compact hyperkähler manifold of $K 3^{[n]}$-type, which is projective if and only if $S$ is projective.

(ii) If $v^{2} \geqslant 2$, there is a Hodge isometry $\lambda_{v}: v^{\perp} \longrightarrow H^{2}\left(M_{v}, \mathbb{Z}\right)$.

The case $v^{2}=0$ was already treated in [PT17]; in this case, there is a Hodge isometry

$$
\lambda_{v}: v^{\perp} / \mathbb{Z} v \longrightarrow H^{2}\left(M_{v}, \mathbb{Z}\right),
$$

and there is no need to suppose that $M_{v}(S, \omega)$ is a $b_{2}$-manifold. For as a consequence [PT17, Theorem 1.1], we already know that $M_{v}(S, \omega)$ is a K3 surface.

The proof of Theorem 1.13 is an application of general results about compact, connected complex $b_{2}$-manifolds which are holomorphically symplectic and a limit of compact hyperkähler manifolds. The starting point is a result originally due to Guan (see Proposition 1.21) stating that if $X$ is a compact, connected holomorphically symplectic $b_{2}$-manifold which is deformation equivalent to a compact hyperkähler manifold, then on $H^{2}(X, \mathbb{Z})$, there is a non-degenerate quadratic form $q_{X}$ of signature $\left(3, b_{2}(X)-3\right)$, and the local Torelli theorem holds.

This result is a generalization of the well-known analogue for compact hyperkähler manifolds proved by Beauville in [Bea83]. By the local Torelli theorem, we mean that the period map is locally a biholomorphism (as in the case of compact hyperkähler manifolds) onto the period domain. We will recall the definition of the Beauville-Bogomolov form and the local Torelli theorem in Section 2.

Remark 1.17. In [PT17], we proved (see Theorem 1.1 there) that if $M$ is a moduli space of slopestable coherent sheaves over a non-projective K3 surface (verifying all the hypotheses of Theorem 1.13), then on $H^{2}(M, \mathbb{Z})$, there is a non-degenerate quadratic form of signature $\left(3, b_{2}(M)-3\right)$. This is proved without assuming that $M$ is a $b_{2}$-manifold. In [PT17], we did not prove the local Torelli theorem, which we find here as a consequence of the assumption that $M$ is a $b_{2}$-manifold.

In Section 2, we consider compact, connected holomorphically symplectic $b_{2}$-manifolds which are not only deformation equivalent to a compact hyperkähler manifold but moreover a limit of compact hyperkähler manifolds. The main result of Section 2 is the following.

THEOREM 1.18. Let $X$ be a compact, connected holomorphically symplectic manifold verifying the $\partial \bar{\partial}$-lemma for 2-forms. If $X$ is a limit of compact hyperkähler manifolds, then $X$ is bimeromorphic to a compact hyperkähler manifold (hence, it is in the Fujiki class $\mathcal{C}$ ).

Using Theorem 1.18, we will show that the same conclusion holds when $X$ is a compact, connected holomorphically symplectic $b_{2}$-manifold which verifies conditions which are more general than the $\partial \bar{\partial}$-lemma for 2 -forms (see Proposition 2.7 for the precise statement). As we will see, if a moduli space $M$ verifies the hypotheses of Theorem 1.13 and is a $b_{2}$-manifold, then it is a compact, connected holomorphically symplectic manifold which is a limit of compact hyperkähler manifolds and which verifies the conditions in Proposition 2.7. In particular, this will allow us to conclude that such moduli spaces are all bimeromorphic to a compact hyperkähler manifold. 


\section{A. Perego}

The proof of Theorem 1.18 is based on a well-known strategy already used by Siu in [Siu83] to show that all K3 surfaces are Kähler, and by Huybrechts in [Huy99] to show that nonseparated, marked, compact hyperkähler manifolds are in fact bimeromorphic. More precisely, if $\Lambda$ is a lattice, we say that a compact complex manifold $X$ carries a $\Lambda$-marking if on $H^{2}(X, \mathbb{Z})$, there is a non-degenerate quadratic form and there is an isometry $\phi: H^{2}(X, \mathbb{Z}) \longrightarrow \Lambda$. The pair $(X, \phi)$ is called a $\Lambda$-marked manifold.

The set of (equivalence classes of) $\Lambda$-marked manifolds is denoted by $\mathcal{M}_{\Lambda}$. As a consequence of the local Torelli theorem, it contains the subset $\mathcal{M}_{\Lambda}^{s}$ of $\Lambda$-marked manifolds $(X, \phi)$ where $X$ is a compact holomorphically symplectic $b_{2}$-manifold which is deformation equivalent to a compact hyperkähler manifold (and whose Beauville-Bogomolov lattice is isometric to $\Lambda$ ). By the local Torelli theorem, we can give $\mathcal{M}_{\Lambda}^{s}$ the structure of a complex space, in which we have a (nonempty) open subset $\mathcal{M}_{\Lambda}^{h k}$ of compact hyperkähler manifolds and a (non-empty) open subset $\mathcal{M}_{\Lambda}^{\partial \bar{\partial}}$ of manifolds verifying the $\partial \bar{\partial}$-lemma for 2-forms. We let $\overline{\mathcal{M}}_{\Lambda}^{h k}$ be the closure of $\mathcal{M}_{\Lambda}^{h k}$ in $\mathcal{M}_{\Lambda}^{s}$.

Theorem 1.18 can be restated by saying that if $(X, \phi) \in \overline{\mathcal{M}}_{\Lambda}^{h k} \cap \mathcal{M}_{\Lambda}^{\partial \bar{\partial}}$, then $X$ is bimeromorphic to a compact hyperkähler manifold. This is the statement we prove; the idea of the proof is that if $(X, \phi) \in \overline{\mathcal{M}}_{\Lambda}^{h k}$, then $(X, \phi)$ is non-separated from a point $(Y, \psi) \in \mathcal{M}_{\Lambda}^{h k}$. A standard argument shows that $X$ and $Y$ have to be bimeromorphic. Theorem 1.18 is just an intermediate result on the way to the Kählerness of the moduli spaces, and it is used in Section 3 to prove that on a compact, connected holomorphically symplectic $b_{2}$-manifold which is a limit of compact hyperkähler manifolds, we can define an analogue of the positive cone of a compact hyperkähler manifold.

Recall that if $X$ is compact hyperkähler and $\mathcal{C}_{X}$ is the cone of real $(1,1)$-classes over which the Beauville-Bogomolov form is strictly positive, the positive cone $\mathcal{C}_{X}^{+}$is the connected component of $\mathcal{C}_{X}$ which contains the Kähler cone of $X$. A result of Huybrechts shows that $\mathcal{C}_{X}^{+}$is contained in (the interior of) the pseudo-effective cone of $X$. Theorem 1.18 is used to prove that on a compact, connected holomorphically symplectic $b_{2}$-manifold $X$ which is a limit of compact hyperkähler manifolds and which is in the Fujiki class $\mathcal{C}$, the intersection of the pseudo-effective cone of $X$ and of $\mathcal{C}_{X}$ (which can be defined as for compact hyperkähler manifolds by the local Torelli theorem) consists of exactly one of the two connected components of $\mathcal{C}_{X}$. This component is the positive cone of $X$, still denoted by $\mathcal{C}_{X}^{+}$. We then prove the following result.

THEOREm 1.19. Let $X$ be a compact, connected holomorphically symplectic manifold in the Fujiki class $\mathcal{C}$ which is a limit of compact hyperkähler manifolds. If there is an $\alpha \in \mathcal{C}_{X}^{+}$such that

(i) $\alpha \cdot C>0$ for every rational curve $C$ on $X$ and

(ii) for every non-zero $\beta \in H^{1,1}(X) \cap H^{2}(X, \mathbb{Z})$, we have $q_{X}(\alpha, \beta) \neq 0$,

then $X$ is compact hyperkähler and $\alpha$ is a Kähler class on $X$.

The proof of this result is based on Theorem 1.18, which gives a bimeromorphism $f: Y--\rightarrow X$ between $X$ and a compact hyperkähler manifold $Y$. Using twistor lines for real $(1,1)$-classes on $X$ (which can be defined similarly to the hyperkähler case thanks to the local Torelli theorem) and a strategy used by Huybrechts for compact hyperkähler manifolds, we show that the conditions on $\alpha$ imply that $f^{*} \alpha$ is a Kähler class on $Y$. An easy argument then shows that $f$ is a biholomorphism and that $\alpha$ is a Kähler class on $X$.

The last part of the paper is devoted to showing that on $M$, a class $\alpha$ as in the statement of 


\section{KÄHLERNESS OF MODULI SPACES}

Theorem 1.19 exists. This is obtained by using the (Hodge) isometry

$$
\lambda_{v}: v^{\perp} \otimes \mathbb{R} \longrightarrow H^{2}(M, \mathbb{R})
$$

(whose existence was proved in [PT17, Section 5.2]) to produce classes in $\mathcal{C}_{M}$. By deforming to a moduli space of slope-stable coherent sheaves on a projective K3 surface, and by using a classical construction of ample line bundles on $M$ in this case (starting from an ample line bundle on $S$ ), we will show that a class as in Theorem 1.19 exists, concluding the proof of Theorem 1.13.

\subsection{The Beauville-Bogomolov form and the local Torelli theorem}

The starting point of the proof of Theorem 1.13 is the local Torelli theorem, which is due to Guan in this generality. We will not prove it here (the proof can be found in [Gua95b]), but we recall the definition of $q_{X}$ and the local Torelli theorem.

1.3.1 The Beauville-Bogomolov form on $H^{2}(X, \mathbb{C})$. Let $X$ be a compact, connected holomorphically symplectic manifold of complex dimension $2 n$. The Beauville-Bogomolov form of $X$ is a quadratic form on $H^{2}(X, \mathbb{C})$ defined as follows. First, choose a holomorphic symplectic form $\sigma$ on $X$, and assume for simplicity that $\int_{X} \sigma^{n} \wedge \bar{\sigma}^{n}=1$. For every $\alpha \in H^{2}(X, \mathbb{C})$, we let

$$
q_{\sigma}(\alpha):=\frac{n}{2} \int_{X} \alpha^{2} \wedge \sigma^{n-1} \wedge \bar{\sigma}^{n-1}+(1-n) \int_{X} \alpha \wedge \sigma^{n} \wedge \bar{\sigma}^{n-1} \int_{X} \alpha \wedge \sigma^{n-1} \wedge \bar{\sigma}^{n} .
$$

Note that $q_{\sigma}(\sigma+\bar{\sigma})=\left(\int_{X} \sigma^{n} \wedge \bar{\sigma}^{n}\right)^{2}=1$, so $q_{\sigma}$ is non-trivial. The quadratic form $q_{\sigma}$ a priori depends on the choice of $\sigma$.

1.3.2 The period map. Let $X$ be a compact, connected holomorphically symplectic $b_{2^{-}}$ manifold of complex dimension $2 n$, and suppose $h^{2,0}(X)=1$. We let $f: \mathcal{X} \longrightarrow B$ be its Kuranishi family and $0 \in B$ a point such that the fiber $X_{0}$ is isomorphic to $X$. By [Gua95b, Theorem 1 and Remark 1 following it], the manifold $B$ is smooth, and that up to shrinking it, we can even suppose that all the fibers of the Kuranishi family are holomorphically symplectic.

Up to shrinking $B$, for every $b \in B$, the fiber $X_{b}$ of $f$ is a compact, connected holomorphically symplectic $b_{2}$-manifold (since being a $b_{2}$-manifold is an open property). Moreover, again up to shrinking $B$, by the Ehresmann fibration theorem, we can suppose that $\mathcal{X}$ is diffeomorphic to $X \times B$. In particular, this induces a diffeomorphism $u_{b}: X \longrightarrow X_{b}$ for every $b \in B$ and hence an isomorphism of complex vector spaces

$$
u_{b}^{*}: H^{2}\left(X_{b}, \mathbb{C}\right) \longrightarrow H^{2}(X, \mathbb{C}) .
$$

We now let $\mathbb{P}:=\mathbb{P}\left(H^{2}(X, \mathbb{C})\right)$ and

$$
p: B \longrightarrow \mathbb{P}, \quad p(b):=\left[u_{b}^{*}\left(\sigma_{b}\right)\right],
$$

where $\sigma_{b}$ is the holomorphic symplectic form on $X_{b}$ such that $\int_{X_{b}} \sigma_{b}^{n} \wedge \bar{\sigma}_{b}^{n}=1$ (we notice that such a $\sigma_{b}$ is unique as $h^{2,0}(X)=1$, and hence $\left.h^{2,0}\left(X_{b}\right)=1\right)$. The map $p$ is holomorphic and will be called period map of $X$.

We let $Q_{\sigma}$ be the quadric defined by the quadratic form $q_{\sigma}$ in $\mathbb{P}$, that is,

$$
Q_{\sigma}=\left\{\alpha \in \mathbb{P} \mid q_{\sigma}(\alpha)=0\right\},
$$

and $\Omega_{\sigma}$ be the open subset of $Q_{\sigma}$ defined as

$$
\Omega_{\sigma}:=\left\{\alpha \in Q_{\sigma} \mid q_{\sigma}(\alpha+\bar{\alpha})>0\right\} .
$$




\section{A. Perego}

We will need the following.

Proposition 1.20. Let $X$ be a compact, connected holomorphically symplectic $b_{2}$-manifold which is deformation equivalent to a compact hyperkähler manifold $Z$. Then for every $p, q \geqslant 0$ such that $p+q=2$, we have $h^{p, q}(X)=h^{p, q}(Z)$. In particular, $h^{2,0}(X)=1$.

Proof. We let $f: \mathcal{X} \longrightarrow B$ be a smooth and proper family such that there are $0, b \in B$ where $X_{0} \simeq X$ and $X_{b} \simeq Z$. Let $U \subseteq B$ be the subset of those $t \in B$ such that $X_{t}$ is a $b_{2}$-manifold; then $U$ is an open subset of $B$ with respect to the analytic Zariski topology, as the Hodge numbers are upper semi-continuous with respect to the analytic Zariski topology (see [Dem95] and references therein), and $0, b \in U$. The upper semi-continuity of the Hodge numbers then implies that for every $p, q \geqslant 0$ such that $p+q=2$, the Hodge numbers $h^{p, q}\left(X_{t}\right)$ are constant over $U$, and we are done.

The following, known as the local Torelli theorem, is due to Guan in this generality (and to Beauville for hyperkähler manifolds).

Proposition 1.21. Let $X$ be a compact, connected holomorphically symplectic $b_{2}$-manifold which is deformation equivalent to a compact hyperkähler manifold.

(i) The quadratic form $q_{\sigma}$ (and hence $Q_{\sigma}$ and $\Omega_{\sigma}$ ) is independent of $\sigma$ and will therefore be denoted by $q_{X}$ (similarly, we write $Q_{X}$ and $\Omega_{X}$ ).

(ii) Up to a positive rational multiple, the quadratic form $q_{X}$ is a non-degenerate quadratic form on $H^{2}(X, \mathbb{Z})$ of signature $\left(3, b_{2}(X)-3\right)$.

(iii) If $B$ is the base of the Kuranishi family of $X$, we have $p(B) \subseteq \Omega_{X}$, and $p: B \longrightarrow \Omega_{X}$ is a local biholomorphism.

Proof. As $X$ is a holomorphically symplectic $b_{2}$-manifold which is deformation equivalent to a compact hyperkähler manifold $Z$, we know by Proposition 1.20 that $h^{2,0}(X)=1$. Theorem 4 of [Gua95b] (and the ensuing Remark 1 therein) proves that $q_{\sigma}$ is independent of $\sigma$ and that there is a positive rational number $F_{X} \in \mathbb{Q}$ such that for every $\alpha \in H^{2}(X, \mathbb{Z})$, if $2 n$ is the complex dimension of $X$, we have

$$
\int_{X} \alpha^{2 n}=F_{X} q_{X}(\alpha)^{n}
$$

The rational number $F_{X}$ will be called the Fujiki constant of $X$; then $F_{X}$ and $q_{X}$ are shown to be topological invariants of $X$ (see the discussion preceding the proof of [Gua95b, Theorem 4]). As $X$ is a deformation of $Z$, which is compact hyperkähler, we have $q_{X}=q_{Z}$, and hence $q_{X}$ is non-degenerate and of signature $\left(3, b_{2}(X)-3\right)$. The fact that the period map $p$ is a local biholomorphism follows as in [Bea83].

Using the non-generate quadratic form $q_{X}$ (of signature $\left(3, b_{2}(X)-3\right)$ ), we let

$$
\mathcal{C}_{X}^{\prime}:=\left\{\alpha \in H^{2}(X, \mathbb{R}) \mid q_{X}(\alpha)>0\right\},
$$

which is an open cone in $H^{2}(X, \mathbb{R})$ having two connected components. Moreover, we let

$$
\widetilde{H}_{\mathbb{R}}^{1,1}(X):=\operatorname{Im}\left(\left\{\alpha \in H^{1,1}(X) \mid d \alpha=0\right\} \longrightarrow H^{2}(X, \mathbb{C})\right) \cap H^{2}(X, \mathbb{R})
$$

and notice that this consists exactly of the de Rham cohomology classes of real $d$-closed $(1,1)$ forms on $X$. We let

$$
\mathcal{C}_{X}:=\mathcal{C}_{X}^{\prime} \cap \widetilde{H}_{\mathbb{R}}^{1,1}(X),
$$

which is an open cone in $\widetilde{H}_{\mathbb{R}}^{1,1}(X)$ having two connected components. 


\section{KÄHLERNESS OF MODULI SPACES}

\section{Limits of compact hyperkähler manifolds}

This section is devoted to proving that every compact, connected holomorphically symplectic $b_{2}$-manifold $X$ which is a limit of compact hyperkähler manifolds is bimeromorphic to a compact hyperkähler manifold; in other words, we prove Theorem 1.18.

The proof is divided into several sections. First, we construct a moduli space $\mathcal{M}_{Z}$ of marked manifolds, and thanks to the local Torelli theorem, we may give it the structure of a (nonseparated) complex space. It will carry a period map to some period domain, which is locally a biholomorphism. Then, we show that each point in the closure of the open subset of $\mathcal{M}_{Z}$ given by compact hyperkähler manifolds is non-separated from a compact hyperkähler manifold. Adapting an argument of Siu (for K3 surfaces) and Huybrechts (for higher-dimensional compact hyperkähler manifolds), we conclude the proof of Theorem 1.18.

\subsection{The moduli space of $\Lambda$-marked manifolds}

In this section, we let $Z$ be a compact hyperkähler manifold, and we write $(\Lambda, q):=\left(H^{2}(Z, \mathbb{Z}), q_{Z}\right)$ for the Beauville-Bogomolov lattice of $Z$. We let $\mathbb{P}_{\Lambda}:=\mathbb{P}(\Lambda \otimes \mathbb{C})$, and inside of it, we let

$$
Q_{\Lambda}:=\left\{\alpha \in \mathbb{P}_{\Lambda} \mid q(\alpha)=0\right\},
$$

which is the quadric defined by $q$, and

$$
\Omega_{\Lambda}:=\left\{\alpha \in Q_{\Lambda} \mid q(\alpha+\bar{\alpha})>0\right\} .
$$

If $X$ is a compact, connected holomorphically symplectic $b_{2}$-manifold which is deformation equivalent to $Z$, by Propositions 1.20 and 1.21 we know that $H^{2}(X, \mathbb{Z})$ carries a non-degenerate quadratic form $q_{X}$ and that there is an isometry $\phi: H^{2}(X, \mathbb{Z}) \longrightarrow \Lambda$. The isometry $\phi$ is called a $\Lambda$-marking on $X$, and the pair $(X, \phi)$ is a $\Lambda$-marked manifold. The set of $\Lambda$-marked manifolds will be denoted by $\mathcal{M}_{Z}^{\prime}$. Moreover, we let $\mathcal{M}_{Z}:=\mathcal{M}_{Z}^{\prime} / \sim$, where $(X, \phi) \sim\left(X^{\prime}, \phi^{\prime}\right)$ if and only if there is a biholomorphism $f: X \longrightarrow X^{\prime}$ such that $\phi \circ f^{*}=\phi^{\prime}$. The set $\mathcal{M}_{Z}$ will be referred to as the moduli space of $\Lambda$-marked manifolds.

We let $\mathcal{M}_{Z}^{h k}$ be the subset of $\mathcal{M}_{Z}$ of equivalence classes of pairs $(X, \phi)$, where $X$ is a compact hyperkähler manifold; it will be called the moduli space of $\Lambda$-marked hyperkähler manifolds. Moreover, we let $\mathcal{M}_{Z}^{\partial \bar{\partial}}$ be the subset of $\mathcal{M}_{Z}$ of equivalence classes of pairs $(X, \phi)$ where $X$ verifies the $\partial \bar{\partial}$-lemma for 2 -forms.

We first show that $\mathcal{M}_{Z}$ has the structure of a complex space (hence justifying the name space we use for it). The following is a generalization of [Huy12, Proposition 4.3], and requires the same proof.

Proposition 2.1. Let $Z$ be a compact hyperkähler manifold and $(\Lambda, q)$ its Beauville-Bogomolov lattice.

(i) For any $(X, \phi) \in \mathcal{M}_{Z}$, there is an inclusion $i_{X}: B \longrightarrow \mathcal{M}_{Z}$, where $B$ is the base of the Kuranishi family of $X$.

(ii) The set $\mathcal{M}_{Z}$ has the structure of a smooth complex space of dimension $b_{2}(Z)-2$.

(iii) The subsets $\mathcal{M}_{Z}^{h k}$ and $\mathcal{M}_{Z}^{\partial \bar{\partial}}$ are Euclidean open subsets of $\mathcal{M}_{Z}$.

Proof. Let $X$ be a compact, connected holomorphically symplectic $b_{2}$-manifold which is deformation equivalent to $Z$, and let $f: \mathcal{X} \longrightarrow B$ be its Kuranishi family. Up to shrinking $B$, we can suppose that it is a complex disk of dimension $b_{2}(X)-2=b_{2}(Z)-2$, and as we have seen before, for every $b \in B$, we can suppose that $X_{b}$ is a compact, connected holomorphically 


\section{A. Perego}

symplectic $b_{2}$-manifold (which is clearly deformation equivalent to $Z$ ). Moreover, we can suppose that $\mathcal{X}$ is diffeomorphic (over $B$ ) to the trivial family $X \times B$ and that we have a diffeomorphism $u_{b}: X \longrightarrow X_{b}$ inducing an isometry $u_{b}^{*}: H^{2}\left(X_{b}, \mathbb{Z}\right) \longrightarrow H^{2}(X, \mathbb{Z})$. We let $\phi_{b}:=\phi \circ u_{b}^{*}$, which is a $\Lambda$-marking on $X_{b}$, for every $b \in B$. It follows that for every $b \in B$, we have $\left(X_{b}, \phi_{b}\right) \in \mathcal{M}_{Z}$, so that we have a map

$$
i_{X}: B \longrightarrow \mathcal{M}_{Z}, \quad i_{X}(b):=\left(X_{b}, \phi_{b}\right) .
$$

We show that $i_{X}$ is an inclusion. Let $b, b^{\prime} \in B$, and suppose $i_{X}(b)=i_{X}\left(b^{\prime}\right)$. This means that $\left(X_{b}, \phi_{b}\right) \sim\left(X_{b^{\prime}}, \phi_{b^{\prime}}\right)$; that is, there is a biholomorphism $f: X_{b} \longrightarrow X_{b^{\prime}}$ such that

$$
f^{*}=\phi_{b}^{-1} \circ \phi_{b^{\prime}}
$$

By the definition of $\phi_{b}$ and $\phi_{b^{\prime}}$, this means that

$$
f^{*}=\left(\phi \circ u_{b}^{*}\right)^{-1} \circ\left(\phi \circ u_{b^{\prime}}^{*}\right)=\left(u_{b}^{*}\right)^{-1} \circ u_{b^{\prime}}^{*} .
$$

Now, let $\sigma_{b}$ and $\sigma_{b^{\prime}}$ be symplectic forms on $X_{b}$ and $X_{b^{\prime}}$, respectively. As $f$ is a biholomorphism, the form $f^{*} \sigma_{b^{\prime}}$ is holomorphic symplectic on $X_{b}$, and hence $\left[u_{b}^{*} \sigma_{b}\right]=\left[u_{b}^{*} f^{*} \sigma_{b^{\prime}}\right]$. But as $f^{*}=\left(u_{b}^{*}\right)^{-1} \circ u_{b^{\prime}}^{*}$, this implies that $\left[u_{b}^{*} \sigma_{b}\right]=\left[u_{b^{\prime}}^{*} \sigma_{b^{\prime}}\right]$. By the definition of the period map of $X$, this means that $p(b)=p\left(b^{\prime}\right)$. But now recall that by point (iii) of Proposition 1.21 , the period map $p: B \longrightarrow \Omega$ is a local biholomorphism: up to shrinking $B$, for $b \neq b^{\prime} \in B$, we have $p(b) \neq p\left(b^{\prime}\right)$. It follows that up to shrinking $B$, the condition $i_{X}(b)=i_{X}\left(b^{\prime}\right)$ implies $b=b^{\prime}$, and $i_{X}$ is an inclusion of $B$ in $\mathcal{M}_{Z}$. This proves point (i) of the statement.

To give $\mathcal{M}_{Z}$ the structure of a complex space, we just need to show that each point of $\mathcal{M}_{Z}$ has a neighborhood having the structure of a complex manifold and that whenever two neighborhoods of this type intersect, the corresponding complex structures glue. If $(X, \phi) \in \mathcal{M}_{Z}$, the previous part of the proof suggests to view $i_{X}(B)$ as a neighborhood $(X, \phi)$ in $\mathcal{M}_{Z}$. Now, let $(X, \phi),\left(X^{\prime}, \phi^{\prime}\right) \in \mathcal{M}_{Z}$, and let $B$ and $B^{\prime}$ be the bases of the Kuranishi families of $X$ and of $X^{\prime}$, respectively. If $i_{X}(B) \cap i_{X}\left(B^{\prime}\right) \neq \emptyset$, then $B \cap B^{\prime}$ is an open subset of $B$ and $B^{\prime}$, over which the Kuranishi families coincide. This allows us to glue the Kuranishi families along $B \cap B^{\prime}$, and hence the complex structures of $i_{X}(B)$ and $i_{X}\left(B^{\prime}\right)$ can be glued in $\mathcal{M}_{Z}$. This shows that $\mathcal{M}_{Z}$ has the structure of a complex space. We notice that as each base $B$ of a Kuranishi family of a compact, connected holomorphically symplectic $b_{2}$-manifold is smooth (see Section 2.2) of dimension $b_{2}(Z)-2$, it follows that $\mathcal{M}_{Z}$ is a smooth complex space, and its dimension is $b_{2}(Z)-2$. This proves point (ii) of the statement.

The fact that $\mathcal{M}_{\Lambda}^{h k}$ and $\mathcal{M}_{Z}^{\partial \bar{\partial}}$ are Euclidean open subsets of $\mathcal{M}_{Z}$ is a consequence of the fact that the Kählerness property and the property of verifying the $\partial \bar{\partial}$-lemma for 2-forms are open properties in the Euclidean topology (for Kählerness, this is [KS60, Theorem 15], and for the $\partial \bar{\partial}$-lemma for 2-forms, this is contained in the Ph.D. thesis of C.-C. Wu, Harvard University, 2006, or see [AT13, Corollary 2.7]).

The complex space $\mathcal{M}_{Z}$ has two connected components, and one can pass from one to the other by mapping $(X, \phi)$ to $(X,-\phi)$.

We now define the period map in this generality. First, if $\phi: H^{2}(X, \mathbb{Z}) \longrightarrow \Lambda$ is an isometry, we let $\phi_{\mathbb{C}}: H^{2}(X, \mathbb{C}) \longrightarrow \Lambda \otimes \mathbb{C}$ be the map induced by $\phi$ by tensoring with $\mathbb{C}$. We then define the map

$$
\pi: \mathcal{M}_{Z} \longrightarrow \mathbb{P}_{\Lambda}, \quad \pi(X, \phi):=\left[\phi_{\mathbb{C}}(\sigma)\right]
$$

where $\sigma$ is a holomorphic symplectic form on $X$ (this is well defined as $h^{2,0}(X)=1$ ). 


\section{KÄHLERNESS OF MODULI SPACES}

Notice that the $\Lambda$-marking $\phi$ induces an isomorphism

$$
\bar{\phi}: \mathbb{P} \longrightarrow \mathbb{P}_{\Lambda}, \quad \bar{\phi}([\alpha]):=\left[\phi_{\mathbb{C}}(\alpha)\right] .
$$

As $\phi$ is an isometry, restricting $\bar{\phi}$ to $\Omega_{X}$, we get an isomorphism $\bar{\phi}: \Omega_{X} \longrightarrow \Omega_{\Lambda}$. If $B$ is the base of the Kuranishi family of $X$, we have $\pi_{\mid i_{X}(B)}=\bar{\phi} \circ p$ : if $b \in B$ and $\sigma_{b}$ is a symplectic form on $X_{b}$, we have

$$
\bar{\phi}(p(b))=\bar{\phi}\left[u_{b}^{*} \sigma_{b}\right]=\left[\phi_{\mathbb{C}}\left(u_{b}^{*} \sigma_{b}\right)\right]=\left[\left(\phi_{b}\right)_{\mathbb{C}}\left(\sigma_{b}\right)\right]=\pi\left(X_{b}, \phi_{b}\right)=i_{X}(b) .
$$

The first two points of the following proposition are just a translation of Proposition 1.21 in this language. For the last point, the surjectivity is [Huy99, Theorem 8.1], and the general injectivity is the global Torelli theorem of Verbistky.

Proposition 2.2. We have the following properties:

(i) The image of $\pi$ is contained in $\Omega_{\Lambda}$.

(ii) The map $\pi$ is a local biholomorphism.

(iii) If $\mathcal{M}_{Z}^{h k, 0}$ is a connected component of $\mathcal{M}_{Z}^{h k}$, the map $\pi_{\mid \mathcal{M}_{Z}^{h k, 0}}$ is surjective and generically injective.

Now, we let $\overline{\mathcal{M}}_{Z}^{h k}$ be the closure of $\mathcal{M}_{Z}^{h k}$ in $\mathcal{M}_{Z}$. Using this formalism, we can state Theorem 1.18 in an equivalent way.

Proposition 2.3. If $(X, \phi) \in \overline{\mathcal{M}}_{Z}^{h k} \cap \mathcal{M}_{Z}^{\partial \bar{\partial}}$, then $X$ is bimeromorphic to a compact hyperkähler manifold (hence, it is in the Fujiki class $\mathcal{C}$ ).

This is the statement we will prove in the next sections.

\subsection{Non-separatedness in $\mathcal{M}_{Z}$}

The first result we show is the following.

Proposition 2.4. Let $(X, \phi) \in \overline{\mathcal{M}}_{Z}^{h k, 0}$. Then there is a $(Y, \psi) \in \mathcal{M}_{Z}^{h k, 0}$ such that $(X, \phi)$ and $(Y, \psi)$ are non-separated in $\mathcal{M}_{Z}$.

Proof. The statement is clear if $(X, \phi) \in \mathcal{M}_{Z}^{h k, 0}$. We then consider $(X, \phi) \in \overline{\mathcal{M}}_{Z}^{h k, 0} \backslash \mathcal{M}_{Z}^{h k, 0}$. We let $p:=\pi(X, \phi) \in \Omega_{\Lambda}$ be the period of $(X, \phi)$. As $\pi_{\mid \mathcal{M}_{Z}^{h k, 0}}$ is surjective, there is a $(Y, \psi) \in \mathcal{M}_{Z}^{h k, 0}$ such that $\pi(Y, \psi)=p$. We show that $(X, \phi)$ and $(Y, \psi)$ are non-separated in $\mathcal{M}_{Z}$. To do so, let $U_{X}$ and $U_{Y}$ be two open neighborhoods of $(X, \phi)$ and $(Y, \psi)$, respectively, in $\mathcal{M}_{Z}$. Up to shrinking $U_{X}$ and $U_{Y}$, we can suppose $\pi\left(U_{X}\right)=\pi\left(U_{Y}\right)=: V$. Moreover, by point (iii) of Proposition 1.21, up to shrinking $U_{X}$ and $U_{Y}$, we can suppose that $\pi_{\mid U_{Y}}: U_{Y} \longrightarrow V$ and $\pi_{\mid U_{X}}: U_{X} \longrightarrow V$ are biholomorphisms. Finally, as Kählerness is an open property in the Euclidean topology, up to shrinking $U_{X}$ and $U_{Y}$, we can suppose that $U_{Y} \subseteq \mathcal{M}_{Z}^{h k, 0}$.

Now, as $(X, \phi) \in \overline{\mathcal{M}}_{Z}^{h k, 0}$, there exist a compact hyperkähler manifold $X^{\prime}$ and a marking $\phi^{\prime}$ on $X^{\prime}$ such that $\left(X^{\prime}, \phi^{\prime}\right) \in U_{X} \cap \mathcal{M}_{Z}^{h k, 0}$. We can choose $\left(X^{\prime}, \phi^{\prime}\right)$ to be generic. Let $p^{\prime}:=\pi\left(X^{\prime}, \phi^{\prime}\right) \in V$; as $\pi_{\mid U_{Y}}: U_{Y} \longrightarrow V$ is surjective, there is a $\left(Y^{\prime}, \psi^{\prime}\right) \in U_{Y}$ such that $\pi\left(Y^{\prime}, \psi^{\prime}\right)=p^{\prime}$, and as $U_{Y} \subseteq \mathcal{M}_{Z}^{h k, 0}$, we have that $Y^{\prime}$ is a compact hyperkähler manifold. Hence, $\left(X^{\prime}, \phi^{\prime}\right)$ and $\left(Y^{\prime}, \psi^{\prime}\right)$ are two generic points in $\mathcal{M}_{Z}^{h k, 0}$. By point (iii) of Proposition 2.2, we then have $\left(X^{\prime}, \phi^{\prime}\right)=\left(Y^{\prime}, \psi^{\prime}\right)$ in $\mathcal{M}_{Z}$, so that $U_{X} \cap U_{Y} \neq \emptyset$, and we are done.

This result will be the starting point of the proof of Proposition 2.3. 


\section{A. Perego}

\subsection{The proof of Theorem 1.18}

We now prove a key result in the proof of Proposition 2.3.

Lemma 2.5. Let $B$ be a connected complex manifold, let $s \in B$, and let $\mathcal{X} \longrightarrow B$ and $\mathcal{Y} \longrightarrow B$ be two smooth, proper families. We suppose that

(i) for every $b \in B$, the fiber $Y_{b}$ is a compact hyperkähler manifold with a $\Lambda$-marking $\psi_{b}$;

(ii) for every $b \in B$, the fiber $X_{b}$ is a compact, connected holomorphically symplectic $b_{2}$-manifold deformation equivalent to a compact hyperkähler manifold which has a $\Lambda$-marking $\phi_{b}$;

(iii) there is a $d$-closed real $(1,1)$-form $\alpha_{s}$ on $X_{s}$;

(iv) there is a sequence $\left\{b_{n}\right\}_{n \in \mathbb{N}}$ of points of $B$ converging to $s$ such that the two following properties are verified:

(a) For every $n$, there is a Kähler form $\alpha_{n}$ on $X_{b_{n}}$, and $\left\{\alpha_{n}\right\}_{n \in \mathbb{N}}$ is a sequence converging to $\alpha_{s}$.

(b) There is a biholomorphism $f_{b_{n}}: Y_{b_{n}} \longrightarrow X_{b_{n}}$ such that $f_{b_{n}}^{*}=\psi_{b_{n}}^{-1} \circ \phi_{b_{n}}$.

Then $X_{s}$ and $Y_{s}$ are bimeromorphic.

Proof. The existence of the sequence $\left\{b_{n}\right\}$ implies that $\left(X_{s}, \phi_{s}\right)$ and $\left(Y_{s}, \psi_{s}\right)$ are non-separated points in $\mathcal{M}_{Z}$, where $Z$ is a compact hyperkähler manifold among all the $Y_{b}$. We will let $X_{n}:=$ $X_{b_{n}}, Y_{n}:=Y_{b_{n}}, \phi_{n}:=\phi_{b_{n}}, \psi_{n}:=\psi_{b_{n}}$ and $f_{n}:=f_{b_{n}}$. We let $\Gamma_{n}$ be the graph of $f_{n}$ in $X_{n} \times Y_{n}$.

Let $\beta_{s}$ be a Kähler form on $Y_{s}$, and consider a continuous family $\left\{\beta_{t}\right\}_{t \in B}$, where $\beta_{t}$ is a closed $(1,1)$-form on $Y_{t}$. As Kählerness is an open property in the Euclidean topology, there is a Euclidean open neighborhood $U$ of $s$ in $B$ such that for every $t \in U$, the form $\beta_{t}$ is Kähler on $Y_{t}$. In particular, for $n \gg 0$, we have that $\beta_{n}:=\beta_{b_{n}}$ is a Kähler form on $Y_{n}$.

We now compute the volume of $\Gamma_{n}$ in $X_{n} \times Y_{n}$ with respect to the Kähler form $p_{1}^{*} \alpha_{n}+p_{2}^{*} \beta_{n}$, where $p_{1}$ and $p_{2}$ are the projections of $X_{n} \times Y_{n}$ onto $X_{n}$ and $Y_{n}$, respectively. We have

$$
\begin{aligned}
\operatorname{vol}\left(\Gamma_{n}\right) & =\int_{Y_{n}}\left(\beta_{n}+f_{n}^{*} \alpha_{n}\right)^{2 m}=\int_{Y_{n}}\left(\left[\beta_{n}\right]+f_{n}^{*}\left[\alpha_{n}\right]\right)^{2 m} \\
& =\int_{Y_{n}}\left(\left[\beta_{n}\right]+\psi_{n}^{-1} \circ \phi_{n}\left(\left[\alpha_{n}\right]\right)\right)^{2 m},
\end{aligned}
$$

where $2 m$ is the complex dimension of $X$ and $Y$. Taking the limit for $n$ going to infinity, we get

$$
\lim _{n \rightarrow+\infty} \operatorname{vol}\left(\Gamma_{n}\right)=\int_{Y_{s}}\left(\left[\beta_{s}\right]+\psi_{s}^{-1} \circ \phi_{s}\left(\left[\alpha_{s}\right]\right)\right)^{2 m}<+\infty .
$$

Hence, the volumes of the $\Gamma_{n}$ are bounded, so that by the Bishop theorem the cycles $\Gamma_{n}$ converge to a cycle $\Gamma$ of $X_{s} \times Y_{s}$ with the same cohomological properties as the $\Gamma_{n}$. Namely, we have $[\Gamma] \in H^{4 m}\left(X_{s} \times Y_{s}, \mathbb{Z}\right)$, and if $p_{1}$ and $p_{2}$ are the two projections from $X_{s} \times Y_{s}$ onto $X_{s}$ and $Y_{s}$, respectively, we have $p_{1 *}[\Gamma]=\left[X_{s}\right], p_{2 *}[\Gamma]=\left[Y_{s}\right]$ and

$$
[\Gamma]_{*} \gamma:=p_{2 *}\left([\Gamma] \cdot p_{1}^{*} \gamma\right)=\psi_{s}^{-1}\left(\phi_{s}(\gamma)\right)
$$

for every $\gamma \in H^{2}\left(X_{s}, \mathbb{Z}\right)$.

Now, let us split $\Gamma$ into its irreducible components. By the previous properties, we then have two possibilities:

(1) either $\Gamma=Z+\sum_{i} D_{i}$, where $p_{1}: Z \longrightarrow X_{s}$ and $p_{2}: Z \longrightarrow Y_{s}$ are both generically one-to-one;

(2) or $\Gamma=Z_{1}+Z_{2}+\sum_{i} D_{i}$, where $p_{1}: Z_{1} \longrightarrow X_{s}$ and $p_{2}: Z_{2} \longrightarrow Y_{s}$ are generically one-to-one, but neither $p_{1}: Z_{2} \longrightarrow Y_{s}$ nor $p_{2}: Z_{1} \longrightarrow X_{s}$ is generically finite. 


\section{KÄHLERNESS OF MODULI SPACES}

In both cases, we have $p_{1 *}\left[D_{i}\right]=p_{2 *}\left[D_{i}\right]=0$. Following the proof of [Huy12, Theorem 4.3], one can show that possibility (2) can be excluded. It follows that $\Gamma=Z+\sum_{i} D_{i}$, where $p_{1}: Z \longrightarrow X_{s}$ and $p_{2}: Z \longrightarrow Y_{s}$ are generically one-to-one. In conclusion, $X_{s}$ and $Y_{s}$ are bimeromorphic.

A consequence of the previous lemma is the following.

Proposition 2.6. Let $B$ be a connected complex manifold and $\mathcal{X} \longrightarrow B$ and $\mathcal{Y} \longrightarrow B$ be two smooth, proper families verifying the following properties:

(i) For every $b \in B$, the fiber $Y_{b}$ is a compact hyperkähler manifold with a $\Lambda$-marking $\psi_{b}$.

(ii) For every $b \in B$, the fiber $X_{b}$ is a compact, connected holomorphically symplectic manifold verifying the $\partial \bar{\partial}$-lemma for 2 -forms, which is deformation equivalent to a compact hyperkähler manifold, and which has a $\Lambda$-marking $\phi_{b}$.

(iii) There is a $b \in B$ such that there is a biholomorphism $f_{b}: Y_{b} \longrightarrow X_{b}$ with $f_{b}^{*}=\psi_{b}^{-1} \circ \phi_{b}$.

Let $V$ be the maximal open subset of $B$ containing $b$ and for which there is a $V$-biholomorphism $f_{V}: \mathcal{Y}_{V} \longrightarrow \mathcal{X}_{V}$ (where $\mathcal{Y}_{V}$ and $\mathcal{X}_{V}$ denote the restrictions of the families to $V$ ) extending the biholomorphism $f_{b}$. If for a generic $b^{\prime} \in V$, we have $\operatorname{NS}\left(X_{b^{\prime}}\right)=0$, then $V$ is dense in $B$.

Proof. We let $0 \in B$ and $X:=X_{0}$ and $Y:=Y_{0}$. For every $b \in B$, we have identifications

$$
H^{2}\left(X_{b}, \mathbb{Z}\right) \simeq H^{2}\left(Y_{b}, \mathbb{Z}\right) \simeq H^{2}(X, \mathbb{Z}) \simeq H^{2}(Y, \mathbb{Z}) \simeq \Lambda,
$$

where $\Lambda$ is the Beauville-Bogomolov lattice of $Y$. For every $\alpha \in \Lambda$, we let

$$
S_{\alpha}:=\left\{b \in B \mid \alpha \in \mathrm{NS}\left(Y_{b}\right)\right\} .
$$

Similarly, for every $\alpha \in H^{4 m-2}(Y, \mathbb{Z})$ (where $2 m$ is the complex dimension of $X$ and $Y$ ), we let

$$
S_{\alpha}:=\left\{b \in B \mid \alpha \in H^{2 m-1,2 m-1}\left(Y_{b}\right)\right\} .
$$

The subset $S_{\alpha} \subseteq B$ is a smooth hypersurface of $B$ (see [Huy99, Section 1.16]).

The fact that $V$ is open in $B$ is a consequence of the local Torelli theorem. Notice that if $b^{\prime} \in V$, then the biholomorphism $f_{V}$ induces (by restricting to the fibers over $b^{\prime}$ ) a biholomorphism $f_{b^{\prime}}: Y_{b^{\prime}} \longrightarrow X_{b^{\prime}}$ such that $f_{b^{\prime}}^{*}=\psi_{b^{\prime}}^{-1} \circ \phi_{b^{\prime}}$. Letting $\bar{V}$ be the closure of $V$ in $B$ and $\partial V:=\bar{V} \backslash V$, we claim that

$$
\partial V \subseteq \bigcup_{\alpha \in \Lambda \cup H^{4 m-2}(Y, \mathbb{Z})} S_{\alpha}
$$

In particular, this means that $\partial V$ is contained in a countable union of analytic subvarieties of $B$. It has real codimension at least 2 in $B$; hence, it cannot separate the disjoint open subsets $V$ and $B \backslash \bar{V}$. As $V \neq \emptyset$ (since $b \in V$ ), it follows that $B=\bar{V}$, concluding the proof. In order to show the claim, we show that if $s \in \partial V$, then $Y_{s}$ has either effective divisors or curves (hence, there is an $\alpha \in \Lambda \cup H^{4 m-2}(Y, \mathbb{Z})$ such that $\left.s \in S_{\alpha}\right)$. We proceed by contradiction: we let $s \in \partial V$, and we suppose that $Y_{s}$ has no effective divisors and no curves.

The first thing we prove is that $X_{s}$ and $Y_{s}$ are bimeromorphic, by using Lemma 2.5. Points (i) and (ii) of the statement of Lemma 2.5 are fulfilled (since if $X_{b}$ verifies the $\partial \bar{\partial}$-lemma for 2-forms, then it is a $b_{2}$-manifold). Moreover, as $X_{s}$ verifies the $\partial \bar{\partial}$-lemma for 2 -forms, we see that $H_{\mathbb{R}}^{1,1}(X)$ injects into $H^{2}(X, \mathbb{R})$. As the signature of the Beauville-Bogomolov $q_{X_{s}}$ form is $\left(3, b_{2}\left(X_{s}\right)-3\right)$ by Proposition 1.21, and as $q_{X_{s}}$ is strictly positive on $H^{2,0}(X)$ and $H^{0,2}(X)$, it follows that there is a $d$-closed real $(1,1)$-form $\alpha_{s}$ on $X_{s}$ such that $q_{X_{s}}\left(\left[\alpha_{s}\right]\right)>0$. This shows that point (iii) of Lemma 2.5 is fulfilled. Up to shrinking $B$, for every $b \in B$, we then have a $d$-closed real $(1,1)$-form $\alpha_{b}$, which depends continuously on $b$, such that the $\alpha_{b}$ converge to $\alpha_{s}$ and such that $q_{X_{b}}\left(\left[\alpha_{b}\right]\right)>0$. 


\section{A. Perego}

As $s \in \partial V$ and as for the generic point of $b^{\prime} \in V$, we have $\operatorname{NS}\left(X_{b^{\prime}}\right)=0$, there is a sequence of points $b_{n} \in V$ converging to $s$ such that $\operatorname{NS}\left(X_{b_{n}}\right)=0$. As $X_{b_{n}}$ is biholomorphic to $Y_{b_{n}}$, it is a compact hyperkähler manifold. By [Huy99, Corollary 5.7], this implies that either $\alpha_{b_{n}}$ or $-\alpha_{b_{n}}$ is Kähler. Up to changing the sign of $\alpha_{s}$, we can then suppose that $\alpha_{b_{n}}$ is Kähler on $X_{b_{n}}$, and the sequence $\left\{\alpha_{b_{n}}\right\}_{n \in \mathbb{N}}$ converges to $\alpha_{s}$. In conclusion, even point (iv) of Lemma 2.5 is fulfilled.

We are then in the position to apply Lemma 2.5, which gives us that $X_{s}$ and $Y_{s}$ are bimeromorphic. More precisely, there is a cycle $\Gamma \in H^{4 m}\left(X_{s} \times Y_{s}, \mathbb{Z}\right)$ of the form $\Gamma=Z+\sum_{i} D_{i}$, and if $p_{1}$ and $p_{2}$ are the two projections of $X_{s} \times Y_{s}$ onto $X_{s}$ and $Y_{s}$, respectively, then $p_{1}: Z \longrightarrow X_{s}$ and $p_{2}: Z \longrightarrow Y_{s}$ are generically one-to-one. If we let $D \in H^{4 m}\left(X_{s} \times Y_{s}, \mathbb{Z}\right)$ be any cycle, we define

$$
[D]_{*}: H^{2}\left(X_{s}, \mathbb{Z}\right) \longrightarrow H^{2}\left(Y_{s}, \mathbb{Z}\right), \quad[D]_{*} \gamma:=p_{2 *}\left([D] \cdot p_{1}^{*} \gamma\right) .
$$

As seen in the proof of Lemma 2.5, we have $[\Gamma]_{*}=\psi_{s}^{-1} \circ \phi_{s}$. As $Y_{s}$ is supposed to have neither effective divisors nor curves, the bimeromorphism corresponding to $Z$ is a biholomorphism. It then follows that there is a biholomorphism $f: Y_{s} \longrightarrow X_{s}$ whose graph is $Z$, and $X_{s}$ is Kähler (as $Y_{s}$ is). By [Huy99, Corollary 5.7], the Kähler cone of $Y_{s}$, and hence that of $X_{s}$, is one of the components of $\mathcal{C}_{Y_{s}}$. Moreover, as in [Huy99, Lemma 5.5], for all $i$, we have $\left[D_{i}\right]_{*}=0$, so that $f^{*}=[Z]_{*}=[\Gamma]_{*}$.

We claim that $\Gamma=Z$. Indeed, recall that $\alpha_{s}$ is a Kähler class on $X_{s}$, and let $\gamma_{s}:=f^{*} \alpha_{s}$, which is Kähler on $Y_{s}$ (as $f$ is a biholomorphism). We calculate the volumes of $\Gamma$ and $Z$ on $X_{s} \times Y_{s}$ with respect to the Kähler class $p_{1}^{*} \alpha_{s}+p_{2}^{*} \gamma_{s}$. We have

$$
\begin{aligned}
\operatorname{vol}(\Gamma) & =\operatorname{vol}(Z)+\sum_{i} \operatorname{vol}\left(D_{i}\right)=\int_{Z}\left(p_{1}^{*} \alpha_{s}+p_{2}^{*} \gamma_{s}\right)^{2 m}+\sum_{i} \operatorname{vol}\left(D_{i}\right) \\
& =\int_{Y_{s}}\left([Z]_{*} \alpha_{s}+\gamma_{s}\right)^{2 m}+\sum_{i} \operatorname{vol}\left(D_{i}\right)=\int_{Y_{s}}\left(f^{*} \alpha_{s}+\gamma_{s}\right)^{2 m}+\sum_{i} \operatorname{vol}\left(D_{i}\right) \\
& =\int_{Y_{s}}\left(2 \gamma_{s}\right)^{2 m}+\sum_{i} \operatorname{vol}\left(D_{i}\right),
\end{aligned}
$$

where we have used that $f^{*}=[Z]_{*}$.

Now, consider the sequence $\left\{b_{n}\right\}_{b \in \mathbb{N}}$ of generic points in $V$ converging to $s$, and let $X_{n}:=X_{b_{n}}$, $Y_{n}:=Y_{b_{n}}, f_{n}:=f_{b_{n}}, \phi_{n}:=\phi_{b_{n}}, \psi_{n}:=\psi_{b_{n}}$ and $\alpha_{n}:=\alpha_{b_{n}}$. For each $n$, the graph of $f_{n}$ in $X_{n} \times Y_{n}$ is denoted by $\Gamma_{n}$. We let $\gamma_{n}:=f_{n}^{*} \alpha_{n}$, which is a Kähler class on $Y_{n}$. Calculating the volumes of the $\Gamma_{n}$ with respect to $p_{1}^{*} \alpha_{n}+p_{2}^{*} \gamma_{n}$, we have

$$
\operatorname{vol}\left(\Gamma_{n}\right)=\int_{Y_{n}}\left(\left[\Gamma_{n}\right]_{*} \alpha_{n}+\gamma_{n}\right)^{2 m}=\int_{Y_{n}}\left(f_{n}^{*} \alpha_{n}+\gamma_{n}\right)^{2 m}=\int_{Y_{n}}\left(2 \gamma_{n}\right)^{2 m} .
$$

We then have

$$
\lim _{n \rightarrow+\infty} \operatorname{vol}\left(\Gamma_{n}\right)=\int_{Y_{s}}\left(2 \gamma_{s}\right)^{2 m}=\operatorname{vol}(Z)
$$

But notice that

$$
\operatorname{vol}\left(\Gamma_{n}\right)=\int_{Y_{n}}\left(f_{n}^{*} \alpha_{n}+\gamma_{n}\right)^{2 m}=\int_{Y_{n}}\left(\psi_{n}^{-1}\left(\phi_{n}\left(\alpha_{n}\right)\right)+\gamma_{n}\right)^{2 m},
$$

hence

$$
\lim _{n \rightarrow+\infty} \operatorname{vol}\left(\Gamma_{n}\right)=\int_{Y_{s}}\left(\psi_{s}^{-1}\left(\phi_{s}\left(\alpha_{s}\right)\right)+\gamma_{s}\right)^{2 m}=\int_{Y_{s}}\left([\Gamma]_{*} \alpha_{s}+\gamma_{s}\right)^{2 m}=\operatorname{vol}(\Gamma)
$$




\section{KÄHLERNESS OF MODULI SPACES}

We then conclude that $\operatorname{vol}(\Gamma)=\operatorname{vol}(Z)$, so that $\operatorname{vol}\left(D_{i}\right)=0$ for every $i$, and hence $D_{i}=0$ for every $i$. It follows that $\Gamma=Z$, and hence that $s \in V$. As this contradicts the fact that $s \in \partial V$, we conclude that if $s \in \partial V$ on $Y_{s}$, there are either effective divisors or curves, and we are done.

We are now able to prove Proposition 2.3.

Proof of Proposition 2.3. As $(X, \phi) \in \overline{\mathcal{M}}_{Z}^{h k}$, by Proposition 2.4 we know that there is a compact hyperkähler manifold $Y$, together with a marking $\psi$, such that $(X, \phi)$ is non-separated from $(Y, \psi)$ in $\mathcal{M}_{Z}$. Consider $\operatorname{Def}(X)$ and $\operatorname{Def}(Y)$, the bases of the Kuranishi families of $X$ and $Y$, respectively. Up to shrinking them, as the points $(X, \phi)$ and $(Y, \psi)$ are non-separated, the local Torelli theorem allows us to identify them. Hence, the Kuranishi families of $X$ and $Y$ are over the same base $B$, and we suppose that $X$ and $Y$ are over the same point $0 \in B$.

Notice that the fibers $Y_{b}$ of the Kuranishi family of $Y$ are all compact hyperkähler manifolds, and they each come with a marking $\psi_{b}$. As verifying the $\partial \bar{\partial}$-lemma for 2 -forms is an open property, all the fibers $X_{b}$ of the Kuranishi family of $X$ are compact, connected holomorphically symplectic manifolds verifying the $\partial \bar{\partial}$-lemma for 2 -form, and they are all deformation equivalent to the compact hyperkähler manifold $Z$.

The non-separatedness implies that there is a $b \in B$ such that $X_{b}$ and $Y_{b}$ are biholomorphic under a biholomorphism $f_{b}$ such that $f_{b}^{*}=\psi_{b}^{-1} \circ \phi_{b}$. As $B$ is open in $\mathcal{M}_{Z}$, the maximal open subset $V$ of $B$ in the statement of Proposition 2.6 is then open in $\mathcal{M}_{Z}$; the generic point $b^{\prime}$ of $V$ is then such that $\operatorname{NS}\left(X_{b^{\prime}}\right)=0$ (see [Huy99, Section 1.14]). By Proposition 2.6, we then conclude that $\bar{V}=B$. Now, notice that if $0 \in V$, then $X$ and $Y$ are isomorphic, and we are done. If $0 \notin V$, we have $0 \in \partial V$. By Lemma 2.5, it follows that $X$ and $Y$ are bimeromorphic (see the proof of Proposition 2.6).

We conclude this section by proving that even if $(X, \phi)$ is not in $\mathcal{M}_{Z}^{\partial \bar{\partial}}$, the manifold $X$ is bimeromorphic to a compact hyperkähler manifold if some further conditions are verified.

Proposition 2.7. Let $(X, \phi) \in \overline{\mathcal{M}}_{Z}^{h k}$, and suppose that the two following conditions are verified:

(i) There is a $d$-closed real $(1,1)$-form $\alpha$ on $X$.

(ii) Letting $\operatorname{Def}(X)$ be the base of Kuranishi family of $X$ and $0 \in \operatorname{Def}(X)$ the point over which the fiber of the Kuranishi family is $X$, there is a sequence $\left\{b_{n}\right\}_{n \in \mathbb{N}}$ of points in $\operatorname{Def}(X)$ converging to 0 such that the two following properties are verified:

(a) For every $n \in \mathbb{N}$, the fiber $X_{n}$ of the Kuranishi family of $X$ over $b_{n}$ is a compact hyperkähler manifold.

(b) There is a sequence $\left\{\alpha_{n}\right\}_{n \in \mathbb{N}}$ converging to $\alpha$, where $\alpha_{n}$ is a $d$-closed real $(1,1)$-form on $X_{n}$ such that $q_{X_{n}}\left(\left[\alpha_{n}\right]\right)>0$.

Then $X$ is bimeromorphic to a compact hyperkähler manifold (in particular, it is in the Fujiki $\operatorname{class} \mathcal{C}$ ).

Proof. As $(X, \phi) \in \overline{\mathcal{M}}_{Z}^{h k}$, by Proposition 2.4 there is a $(Y, \psi) \in \mathcal{M}_{Z}^{h k}$ such that $(X, \phi)$ and $(Y, \psi)$ are non-separated in $\mathcal{M}_{Z}$. We let $\mathcal{X} \longrightarrow \operatorname{Def}(X)$ and $\mathcal{Y} \longrightarrow \operatorname{Def}(Y)$ be the two Kuranishi families. As $(X, \phi)$ and $(Y, \psi)$ are non-separated, by the local Torelli theorem, we can identify $\operatorname{Def}(X)=\operatorname{Def}(Y)=: B$. We let $0 \in B$ be the point over which the fibers of the Kuranishi families are $X$ and $Y$, respectively. 


\section{A. Perego}

For every $b \in B$, the fiber $Y_{b}$ over $b$ of the Kuranishi family of $Y$ is a compact hyperkähler manifold, equipped with a marking $\psi_{b}$. The fiber $X_{b}$ of the Kuranishi family of $X$ is a compact, connected holomorphically symplectic $b_{2}$-manifold which is deformation equivalent to $Z$, equipped with a marking $\phi_{b}$. Moreover, on $X=X_{0}$ we have a $d$-closed real $(1,1)$-form $\alpha$. Hence, points (i), (ii) and (iii) of Lemma 2.5 are fulfilled (for $s=0$ ). We claim that point (iv) is fulfilled too; this implies that $X=X_{0}$ and $Y=Y_{0}$ are bimeromorphic, concluding the proof.

To prove the claim, consider the sequence $\left\{b_{n}\right\}_{n \in \mathbb{N}}$ of points of $B$ given in the statement. For every $n \in \mathbb{N}$, we let $Y_{n}:=Y_{b_{n}}, \phi_{n}:=\phi_{b_{n}}$ and $\psi_{n}:=\psi_{b_{n}}$. We notice that $\left(X_{n}, \phi_{n}\right)$ and $\left(Y_{n}, \psi_{n}\right)$ are two non-separated points in $\mathcal{M}_{Z}^{h k}$. Indeed, up to shrinking $B$, we can suppose that the period map restricted to $B$ is a biholomorphism. The periods of $\left(X_{n}, \phi_{n}\right)$ and $\left(Y_{n}, \psi_{n}\right)$ are the same; one can then proceed as in the proof of Proposition 2.4 to show that $\left(X_{n}, \phi_{n}\right)$ and $\left(Y_{n}, \psi_{n}\right)$ are non-separated points in $\mathcal{M}_{Z}^{h k}$.

We let $\mathcal{X}_{n} \longrightarrow \operatorname{Def}\left(X_{n}\right)$ and $\mathcal{Y}_{n} \longrightarrow \operatorname{Def}\left(Y_{n}\right)$ be the Kuranishi families of $X_{n}$ and $Y_{n}$, respectively. By the universality of the Kuranishi family, we can identify $\operatorname{Def}\left(X_{n}\right)$ and $\operatorname{Def}\left(Y_{n}\right)$ with open neighborhoods of $b_{n}$ in $B$, and as the points $\left(X_{n}, \phi_{n}\right)$ and $\left(Y_{n}, \psi_{n}\right)$ are non-separated, by the local Torelli theorem we can identify $\operatorname{Def}\left(X_{n}\right)$ and $\operatorname{Def}\left(Y_{n}\right)$. Hence, we have $\operatorname{Def}\left(X_{n}\right)=$ $\operatorname{Def}\left(Y_{n}\right)=: U_{n} \subseteq B$, where $U_{n}$ is an open neighborhood of $b_{n}$ in $B$. Moreover, $\mathcal{X}_{n}$ (respectively, $\mathcal{Y}_{n}$ ) is the restriction of $\mathcal{X}$ (respectively, $\mathcal{Y}$ ) to $U_{n}$.

All the fibers of the Kuranishi families of $Y_{n}$ and $X_{n}$ are compact hyperkähler and come with a marking. In particular, all the fibers of the Kuranishi family of $X_{n}$ are compact, connected holomorphically symplectic manifolds verifying the $\partial \bar{\partial}$-lemma for 2 -forms. As $\left(X_{n}, \phi_{n}\right)$ and $\left(Y_{n}, \psi_{n}\right)$ are non-separated in $\mathcal{M}_{Z}$, there is a point $b_{n}^{\prime} \in U_{n}$ for which there is a biholomorphism $f_{b_{n}^{\prime}}: Y_{b_{n}^{\prime}} \longrightarrow X_{b_{n}^{\prime}}$ such that $f_{b_{n}^{\prime}}^{*}=\psi_{b_{n}^{\prime}} \circ \phi_{b_{n}^{\prime}}$. We let $V_{n} \subseteq U_{n}$ be the maximal open subset of $U_{n}$ containing $b_{n}^{\prime}$ and for which there is a $V_{n}$-biholomorphism $f_{v_{n}}: \mathcal{Y}_{V_{n}} \longrightarrow \mathcal{X}_{V_{n}}$ extending $f_{b_{n}^{\prime}}$. Then $V_{n}$ is open in $U_{n}$, which is open in $\mathcal{M}_{Z}$; for the generic $b^{\prime} \in V_{n}$, we then have $\operatorname{NS}\left(X_{b^{\prime}}\right)=0$, so that by Proposition 2.6, we conclude that $V_{n}$ is dense in $U_{n}$. There is then a sequence $\left\{c_{p, n}\right\}_{p \in \mathbb{N}}$ of generic points of $V_{n}$ converging to $b_{n}$. We let $X_{p, n}:=X_{c_{p, n}}$ and $Y_{p, n}:=Y_{c_{p, n}}$. Moreover, up to restricting $U_{n}$, for every $b \in U_{n}$, there is a $d$-closed real $(1,1)$-form $\alpha_{b}$ on $X_{b}$ which depends continuously on $b$, with $\alpha_{b_{n}}=\alpha_{n}$. We let $\alpha_{m, n}:=\alpha_{c_{m, n}}$; as $q_{X_{n}}\left(\left[\alpha_{n}\right]\right)>0$, we can suppose $q_{X_{m, n}}\left(\left[\alpha_{m, n}\right]\right)>0$ (for $m \gg 0$ ). As the $c_{m, n}$ are generic points, we have $\operatorname{NS}\left(X_{m, n}\right)=0$. By [Huy99, Corollary 5.7], we then have that either $\alpha_{m, n}$ or $-\alpha_{m, n}$ is a Kähler form. Up to changing the sign of $\alpha$ (and hence of $\alpha_{n}$ ), we can then suppose that $\alpha_{m, n}$ is Kähler on $X_{m, n}$.

As for every $n \in \mathbb{N}$, the sequence $\left\{c_{m, n}\right\}$ is contained in $V_{n}$ and converges to $b_{n}$, and as the sequence $\left\{b_{n}\right\}$ converges to 0 , we then produce a sequence $\left\{b_{n}^{\prime}\right\}$ converging to 0 , where $b_{n}^{\prime} \in V_{n}$. As $\left\{\alpha_{m, n}\right\}$ is a sequence of Kähler classes converging to $\alpha_{n}$, and as the sequence $\left\{\alpha_{n}\right\}$ converges to $\alpha$, we then get a sequence $\left\{\alpha_{n}^{\prime}\right\}$ of Kähler classes on $X_{b_{n}^{\prime}}$ converging to $\alpha$. We then see that point (iv) of Lemma 2.5 is fulfilled, and we are done.

\section{Criterion for Kählerness}

We now want to prove a Kählerness criterion for a compact, connected holomorphic symplectic manifold $X$ in the Fujiki class $\mathcal{C}$ which is a limit of compact hyperkähler manifolds. Let us first recall some notation: as $X$ is in the Fujiki class $\mathcal{C}$, the cohomology group $H^{2}(X, \mathbb{C})$ has a Hodge decomposition. In particular, we have

$$
\widetilde{H}_{\mathbb{R}}^{1,1}(X)=H^{2}(X, \mathbb{R}) \cap H^{1,1}(X)=: H^{1,1}(X, \mathbb{R}) .
$$




\section{KÄHLERNESS OF MODULI SPACES}

\subsection{Twistor lines}

If $X$ is a hyperkähler manifold, with any $\alpha \in H^{1,1}(X, \mathbb{R})$, one can associate a twistor line (see [Huy99, Section 1.17]). The same can be done for a compact, connected holomorphically symplectic manifold $X$ in the Fujiki class $\mathcal{C}$, which is deformation equivalent to a compact hyperkähler manifold $Z$ (whose Beauville lattice will be denoted by $(\Lambda, q)$ ). To do so, let $\sigma$ be a holomorphic symplectic form on $X$. The cohomology class of $\sigma$ allows us to define a real plane

$$
P(X):=(\mathbb{C} \cdot \sigma \oplus \mathbb{C} \cdot \bar{\sigma}) \cap H^{2}(X, \mathbb{R})
$$

in $H^{2}(X, \mathbb{R})$, which is independent of $\sigma$ (as $\left.h^{2,0}(X)=1\right)$. If $\alpha \in H^{1,1}(X, \mathbb{R})$, we let

$$
F(\alpha):=P(X) \oplus \mathbb{R} \cdot \alpha,
$$

which is a 3 -dimensional real subspace of $H^{2}(X, \mathbb{R})$, and we let $F(\alpha)_{\mathbb{C}}:=F(\alpha) \otimes \mathbb{C}$.

If $\phi: H^{2}(X, \mathbb{Z}) \longrightarrow \Lambda$ is a $\Lambda$-marking on $X$ (which exists by Proposition 1.21), consider the point $(X, \phi) \in \mathcal{M}_{Z}$. As $X$ is a limit of compact hyperkähler manifolds, we have $(X, \phi) \in \overline{\mathcal{M}}_{Z}^{h k}$. Notice that $F(\alpha)_{\mathbb{C}}$ is a 3 -dimensional linear subspace of $H^{2}(X, \mathbb{C})$; hence, $\phi_{\mathbb{C}}\left(F(\alpha)_{\mathbb{C}}\right)$ is a 3 dimensional subspace of $\Lambda \otimes \mathbb{C}$, and $\mathbb{P}\left(\phi_{\mathbb{C}}\left(F(\alpha)_{\mathbb{C}}\right)\right.$ is a plane in $\mathbb{P}_{\Lambda}$ (here $\phi_{\mathbb{C}}$ is the isomorphism induced by $\phi$ by tensoring with $\mathbb{C}$ ). Hence, $\mathbb{P}\left(\phi_{\mathbb{C}}\left(F(\alpha)_{\mathbb{C}}\right)\right) \cap \Omega_{\Lambda}$ is a curve in $\Omega_{\Lambda}$ passing through $\pi(X, \phi)$.

If $B$ is the base of the Kuranishi family of $X$, the inverse image

$$
T(\alpha):=\pi^{-1}\left(\mathbb{P}\left(\phi_{\mathbb{C}}\left(F(\alpha)_{\mathbb{C}}\right)\right) \cap \Omega_{\Lambda}\right) \cap B
$$

is a curve in $B$, which will be called the twistor line of $\alpha$. The restriction of the Kuranishi family of $X$ to $T(\alpha)$ will be denoted by

$$
\kappa_{\alpha}: \mathcal{X}(\alpha) \longrightarrow T(\alpha)
$$

For every $t \in T(\alpha)$, there is a real $(1,1)$-class $\alpha_{t}$ on the fiber $X_{t}$ of the Kuranishi family of $X$ over $t$, and the sequence $\left\{\alpha_{t}\right\}$ converges to $\alpha$. If $\alpha$ is Kähler, then $T(\alpha) \simeq \mathbb{P}^{1}$, and the class $\alpha_{t}$ is Kähler on $X_{t}$ for every $t \in T(\alpha)$.

\subsection{Cones in $H^{1,1}(X, \mathbb{R})$}

We define $\mathcal{C}_{X}=\left\{\alpha \in H^{1,1}(X, \mathbb{R}) \mid q_{X}(\alpha)>0\right\}$, which is an open cone in $H^{1,1}(X, \mathbb{R})$ having two connected components. If $X$ is Kähler (and hence compact hyperkähler), the Kähler cone $\mathcal{K}_{X}$ of $X$ (that is, the open convex cone of Kähler classes on $X$ ) is contained in one of them; such a component is usually called positive cone of $X$ and denoted by $\mathcal{C}_{X}^{+}$. If $\operatorname{NS}(X)=0$, Corollary 5.7 of [Huy99] gives us that $\mathcal{K}_{X}=\mathcal{C}_{X}^{+}$, a fact that has already been used in the previous sections.

Theorem 1.1 of [Bou01] tells us that if $X$ is compact hyperkähler, then $\alpha \in \mathcal{C}_{X}^{+}$is in the Kähler cone of $X$ if and only if $\int_{C} \alpha>0$ for every rational curve $C$ of $X$. Our aim is to show a similar result for a compact, connected holomorphic symplectic manifold $X$ in the Fujiki class $\mathcal{C}$ which is a limit of compact hyperkähler manifolds. As on such a manifold, the Kähler cone could be empty, we cannot use it to define the positive cone of $X$. Instead, we can use the pseudo-effective cone $\mathcal{E}_{X}$ of $X$, that is, the closed convex cone of classes of positive closed real $(1,1)$-currents on $X$. If $X$ is compact hyperkähler, by [Bou04, Theorem $4.3 \mathrm{i}$ )], we have $\mathcal{C}_{X}^{+} \subseteq \mathcal{E}_{X}$.

Popovici and Ugarte [PU18, Theorem 5.9] showed that if $\mathcal{X} \longrightarrow B$ is a smooth and proper family of sGG manifolds [PU18, Definition 1.2] and $\left\{b_{n}\right\}$ is a sequence of points of $B$ converging to a point $b \in B$, then the limit of the pseudo-effective cones of $X_{b_{n}}$ is contained in $\mathcal{E}_{X_{b}}$, that is, the pseudo-effective cone varies upper-semicontinuously along $B$. As all manifolds in the Fujiki 


\section{A. Perego}

class $\mathcal{C}$ are sGG manifolds [PU18], we conclude that the pseudo-effective cone varies uppersemicontinuously in families of class $\mathcal{C}$ manifolds.

We now prove the following general fact about convex cones in a real finite dimensional vector space.

Lemma 3.1. Let $V$ be a real vector space of finite dimension $n$ and $A, B \subseteq V$ two cones in $V$ such that

(i) the cone $A$ is strictly convex (that is, it does not contain any linear subspace of $V$ ) and closed;

(ii) the cone $B$ is open and has two connected components, each of which is convex;

(iii) for every $a \in B$, we have either $a \in A$ or $-a \in A$.

Then $A \cap B$ is one of the connected components of $B$.

Proof. We first notice that if $B^{+}$and $B^{-}$are the two connected components of $B$, then if $B^{+} \subseteq A$, we have $B^{+}=B \cap A$. Indeed, if $b^{\prime} \in B^{-} \cap A$, then $-b^{\prime} \in B^{+} \subseteq A$. It follows that $b^{\prime},-b^{\prime} \in A$, which is not possible as $A$ is a strictly convex cone. We are left to prove that there is a connected component of $B$ which is contained in $A$. To do so, let $b_{0} \in B \cap A$, and let $B^{+}$be the connected component of $B$ which contains $b_{0}$. We show that if $b_{1} \in B^{+}$, then $b_{1} \in A$.

Consider the segment $\left[b_{0}, b_{1}\right]:=\left\{b_{t}:=(1-t) b_{0}+t b_{1} \mid t \in[0,1]\right\}$. Suppose $b_{1} \notin A$. We have to find a contradiction. First, notice that as $b_{1} \notin A$, there is a $t \in[0,1)$ such that $b_{t} \notin A$; indeed, if for every $t \in[0,1)$, we had $b_{t} \in A$, then as $A$ is closed, we would have $b_{1} \in A$. As $b_{t}$ and $b_{1}$ are not in $A$, for every $s \in[t, 1]$ we have $b_{s} \notin A$; indeed, as $b_{t}, b_{1} \notin A$ but $b_{t}, b_{1} \in B$, we have $-b_{t},-b_{1} \in A$. As $A$ is convex, the segment $\left[-b_{t},-b_{1}\right]$ (whose elements are the $-b_{s}$ for $s \in[t, 1]$ ) is contained in $A$. But this means that $b_{s} \notin A$ as $A$ is a strictly convex cone. The set of those $t \in[0,1]$ for which $b_{t} \notin A$ has an infimum $t_{0} \in[0,1]$. Hence, for every $t<t_{0}$, we have $b_{t} \in A$, and for every $t>t_{0}$, we have $b_{t} \notin A$. As $b_{t} \in B$, this implies that $-b_{t} \in A$ for every $t>t_{0}$. But as $A$ is closed, these conditions give $b_{t_{0}} \in A$ (as $b_{t} \in A$ for every $t<t_{0}$ ) and $-b_{t_{0}} \in A$ (as $-b_{t} \in A$ for every $\left.t>t_{0}\right)$. As $A$ is a strictly convex cone, we get a contradiction.

Lemma 3.1 will be used in the proof of the following.

Lemma 3.2. Let $X$ be a compact, connected holomorphic symplectic manifold in the Fujiki class $\mathcal{C}$ which is a limit of compact hyperkähler manifolds. Then $\mathcal{C}_{X} \cap \mathcal{E}_{X}$ consists of exactly one connected component of $\mathcal{C}_{X}$.

Proof. The pseudo-effective cone $\mathcal{E}_{X}$ is strictly convex and closed in $H^{1,1}(X, \mathbb{R})$. The cone $\mathcal{C}_{X}$ is open and has two connected components, each of which is convex. We show that if $\alpha \in \mathcal{C}_{X}$, then either $\alpha \in \mathcal{E}_{X}$ or $-\alpha \in \mathcal{E}_{X}$ (which, in particular, implies that $\mathcal{C}_{X} \cap \mathcal{E}_{X} \neq \emptyset$ ). Once this is done, the statement follows from Lemma 3.1.

Fix a compact hyperkähler manifold $Z$ which is deformation equivalent to $X$, and let $(\Lambda, q)$ be its Beauville-Bogomolov lattice. Moreover, let $\alpha \in \mathcal{C}_{X}$, and consider a $\Lambda$-marking $\phi$ on $X$ (whose existence comes from Proposition 1.21). As $X$ is a limit of compact hyperkähler manifolds, we have $(X, \phi) \in \overline{\mathcal{M}}_{Z}^{h k}$. Let $\mathcal{X} \longrightarrow B$ be the Kuranishi family of $X$, and let 0 be the point of $B$ over which the fiber of $\mathcal{X}$ is $X$. As $X$ is in the Fujiki class $\mathcal{C}$, it is a sGG-manifold. This being an open condition [PU18], up to shrinking $B$, we can suppose that for every $b \in B$, the manifold $X_{b}$ is sGG. Moreover, as $(X, \phi) \in \overline{\mathcal{M}}_{Z}^{h k}$, there is a sequence $\left\{b_{n}\right\}$ of points of $B$ converging to 0 over which the fiber $X_{n}$ is compact hyperkähler, and we can even suppose that $\operatorname{NS}\left(X_{n}\right)=0$ (that 


\section{KÄHLERNESS OF MODULI SPACES}

is, we suppose $X_{n}$ to be generic in $\mathcal{M}_{Z}^{h k}$; hence, it has trivial Néron-Severi group; see [Huy99, Section 1.14]).

Now, let $\widetilde{\alpha}$ be a $d$-closed real $(1,1)$-form on $X$ whose cohomology class is $\alpha$. For every $b$ in a neighborhood of $(X, \phi)$ in $\mathcal{M}_{Z}$, there is a $d$-closed real $(1,1)$-form $\widetilde{\alpha}_{b}$ on $X_{b}$ depending continuously on $b$ and converging to $\widetilde{\alpha}$ (for $b$ converging to 0 ). Letting $\alpha_{b}$ be the cohomology class of $\widetilde{\alpha}_{b}$, as $q_{X}(\alpha)>0$ (since $\alpha \in \mathcal{C}_{X}$ ), up to restricting the neighborhood, we can suppose $q_{X_{b}}\left(\alpha_{b}\right)>0$ for every $b$; that is, $\alpha_{b} \in \mathcal{C}_{X_{b}}$. In particular, for every $n$, we have $\alpha_{n} \in \mathcal{C}_{X_{n}}$, and the limit of the $\alpha_{n}$ is $\alpha$. As $\mathrm{NS}\left(X_{n}\right)=0$, by [Huy99, Corollary 5.7], either $\alpha_{n}$ or $-\alpha_{n}$ is Kähler (for all $n \gg 0$ ). But this means that either $\alpha_{n} \in \mathcal{E}_{X_{n}}$ or $-\alpha_{n} \in \mathcal{E}_{X_{n}}$ (for all $n \gg 0$ ). By [PU18, Theorem 5.9], we then conclude that either $\alpha \in \mathcal{E}_{X}$ or $-\alpha \in \mathcal{E}_{X}$.

The connected component of $\mathcal{C}_{X}$ contained in $\mathcal{E}_{X}$ will be denoted by $\mathcal{C}_{X}^{+}$and called the positive cone of $X$, in analogy with the hyperkähler case.

\subsection{Deformations and Kähler classes}

The first result we prove is the following.

Proposition 3.3. Let $X$ be a compact, connected holomorphic symplectic manifold in the Fujiki class $\mathcal{C}$ which is a limit of compact hyperkähler manifolds. Let $\alpha \in \mathcal{C}_{X}$.

(i) If for every non-zero $\beta \in H^{2}(X, \mathbb{Z})$, we have $q_{X}(\alpha, \beta) \neq 0$, then there is a $t \in T(\alpha)$ such that $X_{t}$ is Kähler and either $\alpha_{t}$ or $-\alpha_{t}$ is a Kähler class on $X_{t}$.

(ii) If moreover $\alpha \in \mathcal{C}_{X}^{+}$, then $\alpha_{t}$ is Kähler.

Proof. Let $Z$ be a compact hyperkähler manifold which is deformation equivalent to $X$, and let $(\Lambda, q)$ be its Beauville-Bogomolov lattice. Fix a marking $\phi$ on $X$ (which exists by Proposition 1.21), and consider the point $(X, \phi) \in \mathcal{M}_{Z}$. As $X$ is a limit of compact hyperkähler manifolds, we have $(X, \phi) \in \overline{\mathcal{M}}_{Z}^{h k}$.

We first show that for a generic $t \in T(\alpha)$, the fiber $X_{t}$ is in $\overline{\mathcal{M}}_{Z}^{h k}$. Let $\mathcal{X} \longrightarrow B$ be the Kuranishi family of $X$, and let 0 be the point of $B$ over which the fiber $X_{0}$ is $X$. As $(X, \phi) \in \overline{\mathcal{M}}_{Z}^{h k}$, as seen in the proof of Lemma 3.2 , there is a sequence $\left\{b_{n}\right\}$ of points of $B$ verifying the following properties:

(1) The sequence $b_{n}$ converges to 0 in $B$.

(2) For every $n$, the fiber $X_{n}$ of $\mathcal{X}$ over $b_{n}$ is compact hyperkähler and $\operatorname{NS}\left(X_{n}\right)=0$.

(3) For every $n$, there is $\alpha_{n} \in \mathcal{C}_{X_{n}}$ such that the sequence $\alpha_{n}$ converges to $\alpha$.

As $\operatorname{NS}\left(X_{n}\right)=0$, up to changing the sign of $\alpha$, and hence of $\alpha_{n}$, we can suppose $\alpha_{n} \in \mathcal{K}_{X_{n}}$ for every $n$. We let $T_{n}$ be the twistor line of $\alpha_{n}$, which is a rational curve in $B$ passing through the point $\left(X_{n}, \phi_{n}\right)$. As $\left(X_{n}, \phi_{n}\right)$ converges to $(X, \phi)$, and as $\alpha_{n}$ converges to $\alpha$, we see that the twistor lines $T_{n}$ converge to $T(\alpha)$. This means that if $t \in T(\alpha)$, there is a sequence $\left\{s_{t, n}\right\}$ of points of $B$ such that

(1) the sequence $\left\{s_{t, n}\right\}$ converges to $t$;

(2) for every $n$, we have $s_{t, n} \in T_{n}$.

As $s_{t, n} \in T_{n}$, and as $T_{n}$ is the twistor line of the Kähler class $\alpha_{n}$, we see that the fiber $X_{s_{t, n}}$ of the twistor family of $\alpha_{n}$ over $s_{t, n}$ is a compact hyperkähler manifold. As $s_{t, n}$ converges to $t$, we then see that $X_{t}$ is a limit of compact hyperkähler manifolds. This means that $\left(X_{t}, \phi_{t}\right) \in \overline{\mathcal{M}}_{Z}^{h k}$. 


\section{A. Perego}

Now, recall that we have supposed $X$ to be in the Fujiki class $\mathcal{C}$; hence, in particular, $X$ verifies the $\partial \bar{\partial}$-lemma for 2 -forms. This property being open, we can suppose $\left(X_{t}, \phi_{t}\right) \in \mathcal{M}_{Z}^{\partial \bar{\partial}}$ for $t$ sufficiently near to 0 . By Proposition 2.3, this implies that $X_{t}$ is bimeromorphic to a compact hyperkähler manifold $Y_{t}$. Now, by hypothesis, we have $q_{X}(\alpha, \beta) \neq 0$ for every non-zero $\beta \in H^{2}(X, \mathbb{Z})$. This implies that $T(\alpha)$ does not intersect at 0 (and hence generically) any of the hypersurfaces $S_{\beta}$; that is, for a generic $t \in T(\alpha)$, the period of $\left(X_{t}, \phi_{t}\right)$ is generic in $\Omega_{\Lambda}$ (recall that $S_{\beta}$ is the smooth hypersurface in $\operatorname{Def}(X)$ given by those $b \in \operatorname{Def}(X)$ such that $\beta \in \operatorname{NS}\left(X_{b}\right)$; see the proof of Proposition 2.6).

As the periods of $\left(X_{t}, \phi_{t}\right)$ and $\left(Y_{t}, \psi_{t}\right)$ are equal, it follows that for a generic $t \in T(\alpha)$, the compact hyperkähler manifold $Y_{t}$ is such that $\mathrm{NS}\left(Y_{t}\right)=0$, so that $X_{t}$ and $Y_{t}$ are biholomorphic. It follows that $X_{t}$ is compact hyperkähler and that $\mathcal{K}_{X_{t}}$ is one of the components of $\mathcal{C}_{X_{t}}$. As $\alpha_{t} \in \mathcal{C}_{X_{t}}$, it follows that either $\alpha_{t}$ or $-\alpha_{t}$ is a Kähler class on $X_{t}$. Let us now suppose, moreover, that the class $\alpha$ is even pseudo-effective and that $\alpha_{t}$ is not a Kähler class. By what we just proved, it follows that $-\alpha_{t}$ is Kähler for generic $t \in T(\alpha)$. As $\mathcal{K}_{X_{t}}$ is contained in $\mathcal{E}_{X_{t}}$, we then have a family $-\alpha_{t}$ of pseudo-effective classes converging to $-\alpha$. Now, by [PU18, Theorem 5.9] (which we can apply as by the previous part of the proof, the family $\mathcal{X}(\alpha) \longrightarrow T(\alpha)$ is a family of manifolds in the Fujiki class $\mathcal{C}$, and hence of sGG manifolds), a limit of pseudo-effective classes along the family $\mathcal{X}(\alpha)$ is a pseudo-effective class on $X$. This means that $-\alpha$ is a pseudo-effective class on $X$. As by hypothesis, $\alpha$ is pseudo-effective too, it follows that $\alpha=0$, which is not possible as $q_{X}(\alpha)>0$, concluding the proof.

Remark 3.4. The proof of Proposition 3.3 shows that the statement holds for a generic $t \in T(\alpha)$ sufficiently near to 0 .

We now use Proposition 3.3 to show the following, which is an improved version of Proposition 2.3.

Proposition 3.5. Let $X$ be a compact holomorphic symplectic manifold in the Fujiki class $\mathcal{C}$ which is a limit of compact hyperkähler manifolds, and let $\alpha \in \mathcal{C}_{X}^{+}$be such that $q_{X}(\alpha, \beta) \neq 0$ for every non-zero $\beta \in H^{2}(X, \mathbb{Z})$. Then there exist a compact hyperkähler manifold $Y$ and a cycle $\Gamma=Z+\sum_{i} D_{i}$ in $X \times Y$ such that the following properties are verified:

(i) The cycle $Z$ defines a bimeromorphic map between $X$ and $Y$.

(ii) The projections $D_{i} \longrightarrow X$ and $D_{i} \longrightarrow Y$ have positive-dimensional fibers.

(iii) The cycle $\Gamma$ defines a Hodge isometry $[\Gamma]_{*}$ between $H^{2}(X, \mathbb{Z})$ and $H^{2}(Y, \mathbb{Z})$.

(iv) The class $[\Gamma]_{*} \alpha$ is Kähler.

Proof. Consider the family $\kappa_{\alpha}: \mathcal{X}(\alpha) \longrightarrow T(\alpha)$. By Proposition 3.3, we know that for a generic $t \in T(\alpha)$, the fiber $X_{t}$ of $\kappa_{\alpha}$ over $t$ is a compact hyperkähler manifold and that $\alpha_{t}$ is a Kähler class on it.

Let $\mathcal{X}^{\prime} \longrightarrow T\left(\alpha_{t}\right)$ be the twistor family of $\left(X_{t}, \alpha_{t}\right)$, and notice that $\pi(T(\alpha))$ is identified with an open subset of $\pi\left(T\left(\alpha_{t}\right)\right)$ and that for every $s \in T\left(\alpha_{t}\right)$, the fiber $X_{s}^{\prime}$ of $\mathcal{X}^{\prime}$ over $s$ is Kähler. Restricting the twistor family $\mathcal{X}^{\prime}$ to such an open subset, we then find two families $\mathcal{X}(\alpha) \longrightarrow C$ and $\mathcal{X}^{\prime} \longrightarrow C$ over the same base curve, with isomorphic fibers over $t$ such that the fibers of $\mathcal{X}^{\prime}$ are all Kähler. We let $0 \in C$ be the point over which the fiber of $\mathcal{X}$ is $X$, and we let $X^{\prime}$ be the fiber of $\mathcal{X}^{\prime}$ over 0 . Both families are endowed with natural markings $\phi_{s}$ and $\phi_{s}^{\prime}$ for each $s$ such that $\left(\phi_{t}^{\prime}\right)^{-1} \circ \phi_{t}$ is induced by the biholomorphism $X_{t} \simeq X_{t}^{\prime}$. The class $\alpha_{s}^{\prime}:=\left(\phi_{s}^{\prime}\right)^{-1} \circ \phi_{s}\left(\alpha_{s}\right)$ is a Kähler class on $X_{s}^{\prime}$ for every $s \in C$. In particular, the class $\alpha^{\prime}:=\left(\phi_{0}^{\prime}\right)^{-1} \circ \phi_{0}(\alpha)$ is Kähler on $X^{\prime}$. 


\section{KÄHLERNESS OF MODULI SPACES}

Now, we have $\left(X, \phi_{0}\right) \in \overline{\mathcal{M}}_{Z}^{h k}$ and $\left(X^{\prime}, \phi_{0}^{\prime}\right) \in \mathcal{M}_{Z}^{h k}$. Moreover, by hypothesis, $X$ is in the Fujiki class $\mathcal{C}$; hence, it verifies the $\partial \bar{\partial}$-lemma for 2 -forms. This condition being open, we can suppose that this is the case for all $X_{s}$ in the twistor family of $X$ over $C$ (up to restricting $C$ ). The proof of Proposition 2.6 then shows that there is a cycle $\Gamma=Z+\sum_{i} Y_{i}$ on $X \times X^{\prime}$ such that

(1) the cycle $Z$ defines a bimeromorphic map between $X$ and $X^{\prime}$;

(2) the projections $Y_{i} \longrightarrow X$ and $Y_{i} \longrightarrow X^{\prime}$ have positive-dimensional fibers;

(3) the cycle $\Gamma$ defines a Hodge isometry $[\Gamma]_{*}$ between $H^{2}(X, \mathbb{Z})$ and $H^{2}\left(X^{\prime}, \mathbb{Z}\right)$;

(4) we have $[\Gamma]_{*}=\left(\phi_{0}^{\prime}\right)^{-1} \circ \phi_{0}$.

It follows that $[\Gamma]_{*} \alpha=\alpha^{\prime}$, which is Kähler, and we are done.

\subsection{The proof of Theorem 1.19}

We are now ready to prove Theorem 1.19, namely that if $X$ is a compact, connected holomorphically symplectic manifold in the Fujiki class $\mathcal{C}$ which is a limit of compact hyperkähler manifolds, any very general class $\alpha \in \mathcal{C}_{X}^{+}$(that is,$q_{X}(\alpha, \beta) \neq 0$ for every non-zero $\beta \in H^{2}(X, \mathbb{Z})$ ), such that $\alpha \cdot C>0$ for every rational curve $C$ on $X$ is a Kähler class on $X$, and in particular $X$ is Kähler.

Proof of Theorem 1.19. By Proposition 3.5, as $\alpha \in \mathcal{C}_{X}^{+}$is such that $q_{X}(\alpha, \beta) \neq 0$ for every $\beta \in H^{2}(X, \mathbb{Z})$, there exist a compact hyperkähler manifold $Y$ and a cycle $\Gamma=Z+\sum_{i} D_{i}$ in $X \times Y$ such that the following properties are verified:

(1) The cycle $Z$ defines a bimeromorphic map between $X$ and $Y$.

(2) The projections $D_{i} \longrightarrow X$ and $D_{i} \longrightarrow Y$ have positive-dimensional fibers.

(3) The cycle $\Gamma$ defines a Hodge isometry $[\Gamma]_{*}$ between $H^{2}(X, \mathbb{Z})$ and $H^{2}(Y, \mathbb{Z})$.

(4) The class $\alpha^{\prime}:=[\Gamma]_{*} \alpha$ is Kähler on $Y$.

The argument used in the proof of [Huy03, Theorem 2.5] shows that since $[\Gamma]_{*} \alpha$ is a Kähler class on $Y$ and $\alpha \cdot C>0$ for every rational curve $C$ on $X$, all the irreducible components $D_{i}$ of $\Gamma$ which are contracted by the projection $p_{X}$ of $X \times Y$ to $X$ are such that the codimension in $X$ of $p_{X}\left(D_{i}\right)$ is at least 2. By [Huy99, Lemma 2.2], it then follows that the morphisms $\left[D_{i}\right]_{*}: H^{2}(Y, \mathbb{Z}) \longrightarrow H^{2}(X, \mathbb{Z})$ are all trivial. As a consequence, we have $\alpha=[\Gamma]_{*} \alpha^{\prime}=[Z]_{*} \alpha^{\prime}$.

We let $f: Y \rightarrow X$ be a bimeromorphism whose graph is $Z$. As $\alpha^{\prime}$ is Kähler, for every rational curve $C^{\prime}$ in $Y$, we have $\int_{C^{\prime}} \alpha^{\prime}>0$. Notice that $\alpha^{\prime}=f^{*} \alpha$, so that we have $\int_{C} \alpha>0$ and $\int_{C^{\prime}} f^{*} \alpha>0$ for every rational curve $C$ in $X$ and every rational curve $C^{\prime}$ in $Y$. By [Huy03, Proposition 2.1], it follows that $f$ extends to a biholomorphism, and $\alpha$ is then a Kähler class.

\section{Kählerness of moduli spaces of sheaves}

This last section is devoted to the proof of Theorem 1.13. Hence, we let $S$ be a K3 surface and $v \in H^{2 *}(S, \mathbb{Z})$ be of the form $v=(r, \xi, a)$, where $r>0$ and $\xi \in \mathrm{NS}(S)$ are relatively prime. Moreover, we let $\omega$ be a Kähler class on $S$, which we suppose to be $v$-generic.

We want to show that if the moduli space $M:=M_{v}(S, \omega)$ is a $b_{2}$-manifold, then it is Kähler. To do so, we apply Theorem 1.19 to $M$; we then need to prove that $M$ is a compact, connected holomorphically symplectic manifold in the Fujiki class $\mathcal{C}$ which is a limit of compact hyperkähler manifolds, and we need to provide a very general class $\alpha \in \mathcal{C}_{M}^{+}$such that $\alpha \cdot C>0$ for every rational curve $C$ in $M$. 


\section{A. Perego}

We will always assume $v^{2} \geqslant 2$, as the cases $v^{2} \leqslant 0$ are already known: if $v^{2}<-2$, we have $M=\emptyset$; if $v^{2}=-2$, then $M$ is a point; if $v^{2}=0$, then $M$ is a K3 surface by [PT17, Corollary 5.3].

Step A: The moduli space $M$ is a compact, connected holomorphically symplectic $b_{2}$-manifold which is a limit of compact hyperkähler manifolds. The fact that $M$ is a compact, holomorphically symplectic manifold is due to Toma (see [Tom01, Remark 4.5]). The connectedness is given by[PT17, Proposition 4.24]. The fact that $M$ is a $b_{2}$-manifold is one of the hypotheses in the statement of Theorem 1.13 (that we are proving).

We show the following.

Lemma 4.1. The moduli space $M$ is a limit of compact hyperkähler manifolds.

Proof. Let $\mathcal{S} \longrightarrow B$ be the Kuranishi family of the K3 surface $S$, where $B$ is a complex manifold of dimension 20. Let $B_{\xi} \subseteq B$ be the subvariety of $B$ given by those $b \in B$ such that $\xi \in \operatorname{NS}\left(S_{b}\right)$. Similarly, let $B_{\omega} \subseteq B$ be the subvariety of $B$ given by those $b \in B$ such that the class $\omega$ is in $H^{1,1}\left(S_{b}, \mathbb{R}\right)$. Moreover, let $B_{\xi, \omega}:=B_{\xi} \cap B_{\omega}$. Recall that $B_{\xi}$ and $B_{\omega}$ are smooth hypersurfaces of $B$. Moreover, up to replacing $\omega$ with another class in the same $v$-chamber where it lies, we can suppose that $\xi$ and $\omega$ are linearly independent. Then $B_{\xi}$ and $B_{\omega}$ intersect transversally, so that $B_{\xi, \omega}$ is a smooth analytic subset of $B$ of positive dimension (see [Huy99, Section 1.16]). By [Huy99, Theorem 3.5], the subset $B_{\xi, \omega}^{p}$ of $B_{\xi, \omega}$ given by those $b$ such that $S_{b}$ is projective is dense in $B_{\xi, \omega}$.

We now consider the restriction $\mathcal{S}^{\prime}:=\mathcal{S}_{\mid B_{\xi, \omega}}$, together with a morphism $\mathcal{S}^{\prime} \longrightarrow B_{\xi, \omega}$. We suppose $0 \in B_{\xi, \omega}$ to be such that $S_{0} \simeq S$. Consider the family $\mathcal{S}^{\prime} \longrightarrow B_{\xi, \omega}$ (which is a smooth and proper family of compact Kähler surfaces) and the Hodge bundle $E^{1,1}$ (whose fiber over a point $b \in B_{\xi, \omega}$ is isomorphic to $H^{1,1}\left(S_{b}\right)$ ), together with a morphism $p: E^{1,1} \longrightarrow B_{\xi, \omega}$. Inside $E^{1,1}$, we define the relative Kähler cone $\mathcal{K}$ as the subset of $E^{1,1}$ such that $\mathcal{K}_{b}:=\mathcal{K} \cap p^{-1}(b)$ is the complexified Kähler cone of $S_{b}$ (the open and convex cone in $H^{1,1}\left(S_{b}\right)$ given by the complexified Kähler classes, that is, classes whose imaginary part is a Kähler class).

As a consequence of [KS60, Theorem 15], the relative Kähler cone $\mathcal{K}$ is open in the total space of the vector bundle $E^{1,1}$ with respect to the Euclidean topology (see [Mag12, Proposition 2.1]). Now, recall that $\omega$ is a Kähler class on $S$; that is, $\omega$ defines an element in $i \omega \in \mathcal{K}_{0}$. Consider the constant section $s_{\omega}: B_{\xi, \omega} \longrightarrow E^{1,1}$ sending $b \in B_{\xi, \omega}$ to $i \omega$ (which is in $E^{1,1}$ since $\omega$ is a real $(1,1)$-class on $S_{b}$ for every $\left.b \in B_{\xi, \omega}\right)$. As $\mathcal{K}$ is open in $E^{1,1}$, the intersection of the image of $s_{\omega}$ with $\mathcal{K}$ is open. It follows that there is a Euclidean open subset $D^{\prime} \subseteq B_{\xi, \omega}$ containing 0 and such that for every $b \in D^{\prime}$, we have that $i \omega$ is in the complexified Kähler cone of $S_{b}$, in other words, that $\omega$ is a Kähler class on $S_{b}$. We can then consider the relative moduli space $\mathcal{M} \longrightarrow D^{\prime}$, whose fiber over $b$ is the moduli space $M_{b}=M_{v}\left(S_{b}, \omega\right)$ of $\mu_{\omega}$-stable coherent sheaves on $S_{b}$ whose Mukai vector is $v$.

As the $v$-genericity is an open property in the Euclidean topology, there is an open subset $D$ of $D^{\prime}$ such that for every $b \in D$, the class $\omega$ is $v$-generic. We then consider the restriction $\mathcal{S}_{D}$ of $\mathcal{S}$ to $D$, together with a morphism $\mathcal{S}_{D} \longrightarrow D$. For every $d \in D$, the K3 surface $S_{d}$ comes equipped with a Mukai vector $v=(r, \xi, a)$ and a $v$-generic polarization $\omega$. As a consequence, the restriction of $\mathcal{M}$ to $D$, denoted by $\mathcal{M}_{D}$, is such that for every $d \in D$ the fiber $M_{d}$ is a compact, connected complex manifold (see again [PT17, Proposition 4.24]). The morphism $\mathcal{M}_{D} \longrightarrow D$ is submersive (see, for example, the proof of [PT17, Proposition 4.23]), and its fibers are all compact, connected manifolds. By [Ehr95, Proposition 1], the family $\mathcal{M}_{D} \longrightarrow D$ is a smooth and proper family whose fiber over 0 is $M_{v}(S, \omega)$. 


\section{KÄHLERNESS OF MODULI SPACES}

Now, as $B_{\xi, \omega}^{p}$ is dense in $B_{\xi, \omega}$, it follows that $B_{\xi, \omega}^{p} \cap D$ is dense in $D$. Hence, for the generic point $d \in D$, the fiber $S_{d}$ is a projective K3 surface, and the fiber $M_{d}$ is a compact hyperkähler manifold (see [PT17, Theorem 3.4]). Hence, $M_{v}(S, \omega)$ is a limit of compact hyperkähler manifolds.

Step B: The moduli space $M$ is in the Fujiki class $\mathcal{C}$. In order to show that $M$ is in the Fujiki class $\mathcal{C}$, we make use of Proposition 2.7. To do so, recall that there is a morphism $\lambda_{v}: v^{\perp} \longrightarrow$ $H^{2}(M, \mathbb{Z})$, which is an isometry (since $v^{2} \geqslant 2$ ) with respect to the Mukai pairing on $v^{\perp}$ and the Beauville-Bogomolov form of $M$ (see [PT17, Proposition 5.2]). This remains true if we tensor with $\mathbb{R}$, and we get an isometry $\lambda_{v}: v^{\perp} \otimes \mathbb{R} \longrightarrow H^{2}(M, \mathbb{R})$. We will then construct the desired class $\alpha$ by taking an appropriate element of $v^{\perp} \otimes \mathbb{R}$.

The choice we make is the following: let $m \in \mathbb{N}$ and

$$
\alpha_{m, \omega}:=(-r,-m r \omega, a+m \omega \cdot \xi) \in H^{2 *}(S, \mathbb{R}),
$$

where $\omega \cdot \xi$ is the (real extension of the) intersection product on $S$. First of all, we remark that $\alpha_{m, \omega} \in v^{\perp} \otimes \mathbb{R}$; indeed, by the definition of the Mukai pairing (see Section 1.2), we have

$$
\left(v, \alpha_{m, \omega}\right)_{\mathrm{Muk}}=m r \omega \cdot \xi-r(a+m \omega \cdot \xi)+r a=0,
$$

and hence $\alpha_{m, \omega} \in v^{\perp} \otimes \mathbb{R}$. If we let $\lambda_{v, \mathbb{R}}: v^{\perp} \otimes \mathbb{R} \longrightarrow H^{2}\left(M_{v}, \mathbb{R}\right)$ be the real extension of $\lambda_{v}$, we then define

$$
\alpha:=\lambda_{v}\left(\alpha_{m, \omega}\right) \in H^{2}(M, \mathbb{R}) .
$$

We now show that $q_{M}(\alpha)>0$.

Lemma 4.2. If $m \gg 0$, we have $\alpha \in \mathcal{C}_{M}$.

Proof. We have $q_{M}(\alpha)=q_{M}\left(\lambda_{v}\left(\alpha_{m, \omega}\right)\right)=\left(\alpha_{m, \omega}, \alpha_{m, \omega}\right)_{\mathrm{Muk}}=m^{2} r^{2} \omega^{2}+2 r a+2 m r \omega \cdot \xi$. As $m \gg 0$ and $\omega^{2}>0$ (since $\omega$ is Kähler on $S$ ), we then see that $q_{M}(\alpha)>0$, that is, $\alpha \in \mathcal{C}_{M}$.

We are finally ready to prove the following.

Lemma 4.3. The moduli space $M$ is in the Fujiki class $\mathcal{C}$.

Proof. By Lemma 4.1, there is a marking $\phi$ on $M$ such that $(M, \phi) \in \overline{\mathcal{M}}_{Z}^{h k}$, where $Z=\operatorname{Hilb}^{n}(K 3)$ and $2 n=v^{2}+2$. Let $m \in \mathbb{N}$, and consider a $d$-closed real $(1,1)$-form $\widetilde{\omega}$ on $S$ whose cohomology class is $m r \omega$ and a real 4 -form $\chi$ on $S$ whose cohomology class is $a+m \omega \cdot \xi$ (under a chosen isomorphism $\left.H^{4}(S, \mathbb{R}) \simeq \mathbb{R}\right)$. Let $p_{S}$ and $p_{M}$ be the two projections of $S \times M$ onto $S$ and $M$, respectively, and consider a quasi-universal family $\mathcal{E}$ on $S \times M$ of similitude $\rho$. For every $i$, we let $\gamma_{i}$ be a real $d$-closed $(i, i)$-form on $S \times M$ whose cohomology class is $\operatorname{ch}_{i}(\mathcal{E})$.

By the definition of the morphism $\lambda_{v}$ given in [PT17, Section 5.2], for every class $\alpha=$ $\left(\alpha_{0}, \alpha_{1}, \alpha_{2}\right) \in v^{\perp} \otimes \mathbb{R}$, we have

$$
\lambda_{v}(\alpha)=\frac{1}{\rho}\left[p_{M *}\left(p_{S}^{*}\left(\alpha^{\vee} \cdot \sqrt{t d(S)}\right) \cdot \operatorname{ch}(\mathcal{E})\right)\right]_{2},
$$

where $\alpha^{\vee}=\left(\alpha_{0},-\alpha_{1}, \alpha_{2}\right)$ and $[\cdot]_{2}$ is the component lying in $H^{2}(M, \mathbb{R})$. More explicitly, we have

$$
\rho \alpha=\alpha_{0} \cdot \operatorname{ch}_{3}(\mathcal{E})-p_{S}^{*} \alpha_{1} \cdot \operatorname{ch}_{2}(\mathcal{E})+p_{S}^{*} \alpha_{2} \cdot \operatorname{ch}_{1}(\mathcal{E}) .
$$

We then notice that the real $(1,1)$-form

$$
\widetilde{\alpha}:=-r \cdot \gamma_{3}+p_{S}^{*} \widetilde{\omega} \cdot \gamma_{2}+p_{S}^{*} \chi \cdot \gamma_{1}
$$




\section{A. Perego}

is $d$-closed and its cohomology class is $\rho \alpha$. If $m \gg 0$, by Lemma 4.2 we then conclude that on $M$, there is a $d$-closed real $(1,1)$-form $\widetilde{\alpha}$ such that $q_{M}([\widetilde{\alpha}])>0$.

We now consider the family $\mathcal{M}_{D} \longrightarrow D$ introduced in the proof of Lemma 4.1. The fiber over $0 \in D$ is $M$, and the fiber over a point $b \in D$ is the moduli space $M_{b}:=M_{v}\left(S_{b}, \omega\right)$. The morphism $\lambda_{v}$ can be defined in families by using a relative quasi-universal family, and for every $b \in D$, we have $\alpha \in H^{2}\left(M_{b}, \mathbb{R}\right)$, and $\alpha$ is still the cohomology class of a $d$-closed real $(1,1)$ form. As the Beauville-Bogomolov form is a topological invariant, if $m \gg 0$, we then again have $q_{M_{b}}(\alpha)>0$.

Now, as we saw in the proof of Lemma 4.1 , there is a sequence $\left\{b_{n}\right\}_{n \in \mathbb{N}}$ of points in $D$ converging to 0 and such that $S_{b_{n}}$ is projective. Hence, $M_{b_{n}}$ is a compact hyperkähler manifold. We can then apply Proposition 2.7 to conclude that $M$ is in the Fujiki class $\mathcal{C}$.

Step C: The class $\alpha$ is very general, it lies in $\mathcal{C}_{M}^{+}$, and it is such that $\alpha \cdot C>0$ for every rational curve $C$ of $M$. We start by showing that $\alpha$ is very general in $H^{2}(M, \mathbb{R})$.

LEMma 4.4. If $\omega$ is sufficiently generic, then for every $\beta \in H^{2}(M, \mathbb{Z})$ such that $\beta \neq 0$, we have $q_{M}(\alpha, \beta) \neq 0$.

Proof. As $\beta \in H^{2}(M, \mathbb{Z})$, and as $\lambda_{v}: v^{\perp} \longrightarrow H^{2}(M, \mathbb{Z})$ is an isometry by [PT17, Proposition 5.2], there is a $\gamma \in v^{\perp}$ such that $\beta=\lambda_{v}(\gamma)$. If we write $\gamma=(s, D, b)$, the condition $\gamma \in v^{\perp}$, that is, $(\gamma, v)_{\mathrm{Muk}}=0$, reads $D \cdot \xi=s a+r b$. Again, as $\lambda_{v}$ is an isometry, it follows that

$$
q_{M}(\alpha, \beta)=q_{M}\left(\lambda_{v}\left(\alpha_{m, \omega}\right), \lambda_{v}(\gamma)\right)=\left(\alpha_{m, \omega}, \gamma\right)_{\mathrm{Muk}}=m \omega \cdot(r D-s \xi)+r b-s a .
$$

Suppose $q_{M}(\alpha, \beta)=0$; this is then equivalent to

$$
\omega \cdot(r D-s \xi)=\frac{D \cdot \xi-2 r b}{m},
$$

which means that $\omega$ is on some hyperplane in $H^{2}(S, \mathbb{R})$ associated with $D$. As the family of these hyperplanes is countable (since the family of $D \in H^{2}(S, \mathbb{Z})$ is countable), and as $\omega$ is sufficiently generic, we see that $q_{M}(\alpha, \beta) \neq 0$ for every non-zero $\beta \in \mathrm{NS}(M)$.

Remark 4.5. Recall that a $v$-generic polarization $\omega$ lies in a $v$-chamber, which is an open convex cone of the Kähler cone of $S$ (see [PT17, Section 2.2] for the definition of $v$-chambers). By [PT17, Proposition 3.2], if $\omega$ and $\omega^{\prime}$ are two $v$-generic polarizations lying in the same $v$-chamber, then $M_{v}(S, \omega)=M_{v}\left(S, \omega^{\prime}\right)$; we can then always suppose that $\omega$ is sufficiently generic. By Lemma 4.4, we can then always suppose that $\alpha$ is very general.

Next, we show that $\alpha$ is pseudo-effective.

LEMMA 4.6. If $m \gg 0$, then $\alpha \in \mathcal{C}_{M}^{+}$.

Proof. By Lemma 4.2, we know that $\alpha \in \mathcal{C}_{M}$. By Lemmas 4.1 and 4.3 , we know that $M$ is a limit of compact hyperkähler manifolds and in the Fujiki class $\mathcal{C}$. By Lemma 3.2, in order to show that $\alpha \in \mathcal{C}_{M}^{+}$, we then just need to show that $\alpha \in \mathcal{E}_{M}$. To show this, consider the deformation $\mathcal{M}_{D} \longrightarrow D$ we introduced in the proof of Lemma 4.1. We let $0 \in D$ be the point over which the fiber is $M_{v}(S, \omega)$. For a generic $b \in D$, the fiber is $M_{b}=M_{v}\left(S_{b}, \omega\right)$, where $S_{b}$ is a projective K3 surface; hence, $M_{b}$ is a projective hyperkähler manifold. Notice that $\omega$ is still a $v$-generic Kähler class on $S_{b}$, and the class $\alpha$ is still in $\mathcal{C}_{M_{b}}$, and this for every $b \in D$. We write $\alpha_{0}:=\alpha$.

Now, as shown in [PT17, Remark 3.5], in the same $v$-chamber where $\omega$ lies there is a class of the form $\omega^{\prime}=c_{1}(H)$ for some ample line bundle $H$ on $S_{b}$. We let $\alpha_{1}:=\lambda_{v}\left(\alpha_{m, \omega^{\prime}}\right)$. Moreover, for 


\section{KÄHLERNESS OF MODULI SPACES}

every $t \in[0,1]$, we let $\omega_{t}:=(1-t) \omega+t \omega^{\prime}$, which is a segment contained in the $v$-chamber where $\omega$ and $\omega^{\prime}$ are, and we let $\alpha_{t}:=\lambda_{v}\left(\alpha_{m, \omega_{t}}\right)$. By the linearity of $\lambda_{v}$, we have $\alpha_{t}=(1-t) \alpha_{0}+t \alpha_{1}$, and the image of the map $\alpha:[0,1] \longrightarrow H^{1,1}\left(M_{b}, \mathbb{R}\right)$ defined by letting $\alpha(t):=\alpha_{t}$ is a segment in $\mathcal{C}_{M_{b}}$.

Our aim is to show that $\alpha \in \mathcal{E}_{M}$. As the family $\mathcal{M}_{D} \longrightarrow D$ is a family of manifolds in the Fujiki class $\mathcal{C}$ by the previous paragraph, by [PU18, Theorem 5.9], it is sufficient to show that $\alpha_{0} \in \mathcal{E}_{M_{b}}$ for a generic $b$ around 0 . As for the generic $b$ around 0 , we have that $M_{b}$ is compact hyperkähler, this is equivalent to showing that $\alpha_{0} \in \mathcal{C}_{M_{b}}^{+}$. As $\mathcal{C}_{M_{b}}^{+}$is a convex cone and the segment $\left[\alpha_{0}, \alpha_{1}\right]$ is contained in $\mathcal{C}_{M_{b}}$, to show that $\alpha_{0} \in \mathcal{C}_{M_{b}}^{+}$, it is sufficient to show that $\alpha_{1} \in \mathcal{C}_{M_{b}}^{+}$. But now, as $S_{b}$ is projective, we can use a general construction presented in [HL97]: if $H$ is a $v$-generic ample line bundle on $S_{b}$, we can construct an ample line bundle $L(H)$ on $M_{v}\left(S_{b}, H\right)$, and we have $c_{1}(L(H))=\lambda_{v}\left(\alpha_{m, c_{1}(H)}\right)$. We know that $\omega$ and $c_{1}(H)$ are in the same $v$-chamber, hence $M_{v}\left(S_{b}, \omega\right)=M_{v}\left(S_{b}, H\right)$ (see Remark 4.5); it follows that $\lambda_{v}\left(\alpha_{m, \omega^{\prime}}\right)$ is an ample class on $M_{v}\left(S_{b}, \omega\right)$. It then lies in the Kähler cone of $M_{b}$, and hence in $\mathcal{C}_{M_{b}}^{+}$.

In conclusion, we have shown that up to choosing $m \gg 0$ and $\omega$ sufficiently generic, the class $\alpha$ is a very general class in $\mathcal{C}_{M}^{+}$. We are left to show that $\alpha \cdot C>0$ for every rational curve $C$ in $M$.

LEMma 4.7. If $m \gg 0$ and $\omega$ is sufficiently generic, we have $\alpha \cdot C>0$ for every rational curve $C$ on $M$.

Proof. Let $[C] \in H^{2 n-1,2 n-1}(M, \mathbb{Z})$, and let $\beta_{C} \in \mathrm{NS}(M)$ be the dual of $[C]$, so that $\alpha \cdot C=$ $q_{M}\left(\alpha, \beta_{C}\right)$. We then just need to prove that $q_{M}\left(\alpha, \beta_{C}\right)>0$ for every rational curve $C$ on $M$.

Let $\mathcal{S} \longrightarrow B$ be the Kuranishi family of $S$, and let $0 \in B$ be such that $S_{0}=S$. We let $B_{C}$ be the subset of $B$ of those $b \in B$ such that $\beta_{C} \in \mathrm{NS}\left(S_{b}\right)$; that is, $C$ is a rational curve on $S_{b}$. Consider the intersection $D_{C}:=D \cap B_{C}$, which is an analytic subset of $D$, whose generic point $d$ is such that $S_{d}$ is a projective K3 surface. We let $\mathcal{M}_{C}$ be the restriction of the relative moduli space $\mathcal{M}_{D} \longrightarrow D$ to $D_{C}$ (see Lemma 4.1 for the definition of the family $\mathcal{M}_{D}$ ) and consider the family $\mathcal{M}_{C} \longrightarrow D_{C}$, whose fiber over a point $d \in D_{C}$ is denoted by $M_{d}$. Notice that for every $d \in D_{C}$, we have the class $\alpha \in \mathcal{C}_{M_{d}}$ and the rational curve $C$ on $M_{d}$. As the intersection product of $\alpha$ with $C$ is constant along $D_{C}$, it is sufficient to show that $q_{M_{d}}\left(\alpha, \beta_{C}\right)>0$ for some $d \in D_{C}$.

As $\beta_{C} \in \mathrm{NS}\left(M_{d}\right)$, and as the morphism $\lambda_{v}: v^{\perp} \longrightarrow H^{2}\left(M_{d}, \mathbb{Z}\right)$ is an isometry by [PT17, Proposition 5.2], there is a class $\gamma \in v^{\perp}$ such that $\beta_{C}=\lambda_{v}(\gamma)$. We write $\gamma=(s, \zeta, b)$, where $s, b \in$ $\mathbb{Z}$ and $\zeta \in H^{2}(S, \mathbb{Z})$. Moreover, as $M_{d}$ is a manifold in the Fujiki class $\mathcal{C}$, on $H^{2}(M, \mathbb{Z})$ we have a Hodge decomposition, and the morphism $\lambda_{v}$ is a Hodge isometry (see [PT17, Corollary 5.3]). As $\beta_{C} \in H^{1,1}(M)$, we then need $\gamma \in\left(v^{\perp}\right)^{1,1}$; that is, we have $\zeta \in \operatorname{NS}\left(S_{d}\right)$.

Now, as $\lambda_{v}$ is an isometry, we have

$$
q_{M_{d}}\left(\alpha, \beta_{C}\right)=q_{M_{d}}\left(\lambda_{v}\left(\alpha_{m, \omega}\right), \lambda_{v}(\gamma)\right)=\left(\alpha_{m, \omega}, \gamma\right)_{\mathrm{Muk}} .
$$

It is then sufficient to show that $\left(\alpha_{m, \omega}, \gamma\right)_{\text {Muk }}>0$. By [PT17, Lemma 3.3], there is an ample class $\omega^{\prime}$ on $S_{d}$ which is in the same $v$-chamber of $\omega$ and is such that for every $\eta \in \operatorname{NS}\left(S_{d}\right)$, we have $\omega \cdot \eta=\omega^{\prime} \cdot \eta$. Then we have $\alpha_{m, \omega^{\prime}} \in v^{\perp}$ and $\left(\alpha_{m, \omega}, \gamma\right)_{\text {Muk }}=\left(\alpha_{m, \omega^{\prime}}, \gamma\right)_{\text {Muk }}$. It is then sufficient to show that $\left(\alpha_{m, \omega^{\prime}}, \gamma\right)_{\mathrm{Muk}}>0$. To do so, consider a rational $\omega^{\prime \prime}$ in a neighborhood of $\omega^{\prime}$ in the ample cone of $S_{d}$. Let $p \in \mathbb{N}$, and let $H$ be an ample line bundle on $S_{d}$ such that $p \omega^{\prime \prime}=c_{1}(H)$. As we can choose $m \gg 0$, we can suppose that $m=m^{\prime} p$ for some very big $m^{\prime} \in \mathbb{N}$. As $H$ is $v$-generic, 


\section{A. Perego}

$\lambda_{v}\left(\alpha_{m^{\prime}, c_{1}(H)}\right)$ is the first Chern class of an ample line bundle, so that $\lambda_{v}\left(\alpha_{m^{\prime}, c_{1}(H)}\right) \cdot C>0$. Since

$$
\begin{aligned}
\lambda_{v}\left(\alpha_{m^{\prime}, c_{1}(H)}\right) \cdot C & =q_{M_{d}}\left(\lambda_{v}\left(\alpha_{m^{\prime}, c_{1}(H)}\right), \beta_{C}\right) \\
& =q_{M_{d}}\left(\lambda_{v}\left(\alpha_{m^{\prime}, c_{1}(H)}\right), \lambda_{v}(\gamma)\right)=\left(\alpha_{m^{\prime}, c_{1}(H)}, \gamma\right)_{\mathrm{Muk}},
\end{aligned}
$$

it follows that $\left(\alpha_{m^{\prime}, c_{1}(H)}, \gamma\right)_{\mathrm{Muk}}>0$. But now, notice that

$$
\begin{aligned}
\alpha_{m^{\prime}, c_{1}(H)} & =\left(-r,-m^{\prime} r c_{1}(H), a+m^{\prime} c_{1}(H) \cdot \xi\right) \\
& =\left(-r,-m^{\prime} \operatorname{prc}_{1}(H) / p, a+m^{\prime} p \xi \cdot c_{1}(H) / p\right)=\left(-r,-m r \omega^{\prime \prime}, a+m \omega^{\prime \prime} \cdot \xi\right)=\alpha_{m, \omega^{\prime \prime}} ;
\end{aligned}
$$

hence, $\left(\alpha_{m, \omega^{\prime \prime}}, \gamma\right)_{\mathrm{Muk}}=\left(\alpha_{m^{\prime}, c_{1}(H)}, \gamma\right)_{\mathrm{Muk}}>0$. As this is true for all rational classes $\omega^{\prime \prime}$ in a neighborhood of $\omega^{\prime}$, this implies that $\left(\alpha_{m, \omega^{\prime}}, \gamma\right)_{\mathrm{Muk}} \geqslant 0$. As we saw before, this implies that $\alpha \cdot C \geqslant 0$ for every rational curve $C$ in $M$. But as $\beta_{C} \in \mathrm{NS}(M)$ and $\alpha \cdot C=q_{M}\left(\alpha, \beta_{C}\right)$, and as we know that $\alpha$ is very general by Lemma 4.4, it follows that $\alpha \cdot C \neq 0$. In conclusion, we have $\alpha \cdot C>0$, and we are done.

Step D: The previous steps allow us to complete the proof of Theorem 1.13.

Proof of Theorem 1.13. We consider a K3 surface $S$, a Mukai vector $v=(r, \xi, a)$ such that $r$ and $\xi$ are relatively prime and $v^{2} \geqslant 2$ and a $v$-generic polarization $\omega$. The moduli space $M:=M_{v}(S, \omega)$ is then a compact, connected holomorphically symplectic manifold of dimension $v^{2}+2$, which is deformation equivalent to a Hilbert scheme of points on a projective K3 surface (see [PT17, Theorem 1.1]).

If $M$ is Kähler, then we clearly have $b_{2}(M)=h^{2,0}(M)+h^{1,1}(M)+h^{0,2}(M)$. We are left with the proof of the opposite direction, so we suppose that $M$ is such that $b_{2}(M)=h^{2,0}(M)+$ $h^{1,1}(M)+h^{0,2}(M)$, and we show that $M$ is Kähler. By Lemma 4.1, we know that $M$ is limit of hyperkähler manifolds, and by Lemma 4.3 we know that $M$ is in the Fujiki class $\mathcal{C}$. Moreover, by Lemmas 4.4, 4.6 and 4.7 (and by Remark 4.5), we know that on $M$ there is a real $(1,1)$-class $\alpha$ which lies in the positive cone of $M$, is very general and intersects positively all rational curves on $M$. By Theorem 1.19, it then follows that $M$ is Kähler.

\section{ACKNOWLEDGEMENTS}

The author wishes to thank M. Toma for several useful conversations during the preparation of this work and the anonymous referee for having pointed out a mistake in the first version of the paper.

\section{REFERENCES}

AT13 D. Angella and A. Tomassini, On the $\partial \bar{\partial}$-lemma and Bott-Chern cohomology, Invent. Math. 192 (2013), no. 1, 71-81; doi:10.1007/s00222-012-0406-3.

Bea83 A. Beauville, Variétés Kähleriennes dont la première classe de Chern est nulle, J. Differential Geom. 18 (1983), no. 4, 755-782; doi:10.4310/jdg/1214438181.

Bou01 S. Boucksom, Le cône kählérien d'une variété hyperkählérienne, C. R. Acad. Sci. Paris Sér. I Math. 333 (2001), no. 10, 935-938; doi:10.1016/S0764-4442(01)02158-9.

Bou04 Divisorial Zariski decompositions on compact complex manifolds, Ann. Sci. École Norm. Sup. (4) 37 (2004), no. 1, 45-76; doi:10.1016/j.ansens.2003.04.002.

Cam91 F. Campana, The class $\mathcal{C}$ is not stable by small deformations, Math. Ann. 290 (1991), no. 1, 19-30; doi:10.1007/BF01459236. 


\section{KÄHLERNESS OF MODULI SPACES}

Del68 P. Deligne, Théorème de lefschetz et dégénérescence de suites spectrales, Publ. Math. Inst. Hautes Études Sci. 35 (1968), no. 1, 107-126; doi:10.1007/BF02698925.

Dem95 J.-P. Demailly, Propriétés de semi-continuité de la cohomologie et de la dimension de KodairaIitaka, C. R. Acad. Sci. Paris Sér. I Math. 320 (1995), no. 3, 341-346.

DGMS75 P. Deligne, P. Griffiths, J. Morgan and D. Sullivan, Real homotopy theory of Kähler manifolds, Invent. Math. 29 (1975), no. 3, 245-274; doi:10.1007/BF01389853.

DP04 J.-P. Demailly and M. Paun, Numerical characterization of the Kähler cone of a compact Kähler manifold, Ann. of Math. 159 (2004), no. 3, 1247-1274; doi:10.4007/annals.2004.159.1247.

Ehr95 C. Ehresmann, Les connexions infinitésimales dans un espace fibré différentiable, Séminaire Bourbaki, vol. 1, Exp. No. 24 (Soc. Math. France, Paris, 1995), 153-168.

Gua94 D. Guan, Examples of compact holomorphic symplectic manifolds which admit no Kähler structure, in Geometry and Analysis on Complex Manifolds (World Sci. Publ., River Edge, NJ, 1994), $63-74$.

Gua95a Examples of compact holomorphic symplectic manifolds which are not Kählerian. II, Invent. Math. 121 (1995), no. 1, 135-145; doi:10.1007/BF01884293.

Gua95b—- Examples of compact holomorphic symplectic manifolds which are not Kählerian. III, Internat. J. Math. 6 (1995), no. 5, 709-718; doi:10.1142/S0129167X95000298.

Hir62 H. Hironaka, An example of a non-Kählerian complex-analytic deformation of Kählerian complex structures, Ann. of Math. 75 (1962), no. 2, 190-208; doi:10.2307/1970426.

HL97 D. Huybrechts and M. Lehn, The geometry of moduli spaces of sheaves, Aspects of Mathematics, vol. E31 (Friedr. Vieweg \& Sohn, Braunschweig, 1997); doi:10.1007/978-3-663-11624-0.

Huy99 D. Huybrechts, Compact hyper-Kähler manifolds: basic results, Invent. Math. 135 (1999), no. 1, 63-113; doi:10.1007/s002220050280.

Huy03 , The Kähler cone of a compact hyperkähler manifold, Math. Ann. 326 (2003), no. 3, 499-513; doi:10.1007/s00208-003-0433-x.

Huy12_, A global Torelli theorem for hyperkähler manifolds [after M. Verbitsky], Astérisque 348 (2012), Exp. No. 1040, x, 375-403.

KS60 K. Kodaira and D. C. Spencer, On deformations of complex analytic structures. III. Stability theorems for complex structures, Ann. of Math. 71 (1960), no. 1, 43-76; doi:10.2307/1969879.

Mag12 G. Magnússon, Natural metrics associated to families of compact Kähler manifolds, Ph.D. Thesis, Université de Grenoble, 2012, available at https://tel.archives-ouvertes.fr/ tel-00849096.

Poo86 Y.S. Poon, Compact self-dual manifolds with positive scalar curvature, J. Differential Geom. 24 (1986), no. 1, 97-132; doi:10.4310/jdg/1214440260.

Pop14 D. Popovici, Deformation openness and closedness of various classes of compact complex manifolds; examples, Ann. Sc. Norm. Super. Pisa Cl. Sci. (5) 13 (2014), no. 2, 255-305.

PT17 A. Perego and M. Toma, Moduli spaces of bundles over nonprojective K3 surfaces, Kyoto J. Math. 57 (2017), no. 1, 107-146; doi:10.1215/21562261-3759540.

PU18 D. Popovici and L. Ugarte, Compact complex manifolds with small Gauduchon cone, Proc. London Math. Soc. 116 (2018), no.5, 1161-1186; doi:10.1112/plms.12110.

Siu83 Y.-T. Siu, Every K3 surface is Kähler, Invent. Math. 73 (1983), no. 1, 139-150; doi:10.1007/BF01393829.

Tom01 M. Toma, Compact moduli spaces of stable sheaves over non-algebraic surfaces, Doc. Math. 6 (2001), 11-29.

Arvid Perego perego@dima.unige.it

Dipartimento di Matematica dell'Università di Genova, via Dodecaneso 35, 16146 Genova, Italy 